To Be published by the Astrophysical Journal

Preprint typeset using $\mathrm{LT}_{\mathrm{E}} \mathrm{X}$ style emulateapj v. 6/22/04

\title{
DARK MATTER HALOS AND EVOLUTION OF BARS IN DISK GALAXIES: COLLISIONLESS MODELS REVISITED
}

\author{
Jorge Villa-Vargas $^{1}$, Isaac Shlosman ${ }^{2,3}$ and Clayton Heller ${ }^{4}$ \\ To be published by the Astrophysical Journal
}

\begin{abstract}
We construct and evolve three one-parameter families and one two-parameter family of steady-state models of stellar disks embedded in live dark matter (DM) halos, in order study the dynamical and secular phases of bar evolution. These models are tested against those published in the literature in order to extend them and to include the gaseous component in the follow up paper. Specifically, we are interested in the angular momentum, $J$, redistribution in the disk-halo system during these two evolutionary phases without distinguishing between the resonant and non-resonant effects. We confirm the previous results and quantify for the first time the dual role that the DM halos play in the bar evolution: more centrally concentrated halos dilute the dynamical processes of the initial bar growth, such as the spontaneous bar instability and the vertical buckling instability, and slow down the $J$ transfer, while facilitating it in the secular phase. The rate of $J$ transfer in the disk and the halo is followed up in order to identify sites and times of peak activity in $J$ emission and absorption. Within the corotation radius, $R_{\mathrm{cr}}$, the disk $J$ remains nearly constant in time, as long as $R_{\mathrm{cr}}$ stays within the disk - a sign that the lost angular momentum to the outer disk and the halo is being compensated by an influx of fresh $J$ due to the outward motion of $R_{\mathrm{cr}}$. We demonstrate that this is feasible as long as the bar slowdown dominates the loss of $J$ inside $R_{\mathrm{cr}}$. Next, we find that in some models the bar pattern speed stalls for prolonged time periods, i.e., the bar exhibits a constant rate of tumbling when $R_{\mathrm{cr}}$ is located outside the disk. This phenomenon appears concurrent with the near absence of $J$ transfer between the disk and the halo, and is associated with the halo emitting $J$ at the corotation resonance and absorbing it at the inner Lindblad resonance. Furthermore, we confirm that stellar bars generally display the corotation-to-bar size ratios in the range of $\sim 1-1.4$, but only between the times of the first buckling and $R_{\mathrm{cr}}$ leaving the disk. Hence, the corotation-to-disk size ratio emerges as an important dynamic discriminator between various stages of barred disk evolution. Finally, we analyze a number of correlations between the basic parameters of a barred disk and a halo, some already reported in the literature and some new.
\end{abstract}

Subject headings: galaxies: evolution - galaxies: halos - galaxies: kinematics and dynamics - galaxies: spiral — galaxies: structure - stellar dynamics — cosmology: dark matter

\section{INTRODUCTION}

Bars are expected to have a profound effect on disk galaxy evolution because they constitute a major departure from an axial symmetry and hence facilitate angular momentum and mass redistribution. Despite prolonged and focused investigation of bar formation and evolution many theoretical and observational issues remain unresolved. Among these are the origin of bars in the universe - spontaneous or tidally induced, evolution of bar fractions with redshifts, and theoretical predictions of bar pattern speeds. The intricacies of the bar growth or decay with time are obscure - how do bars capture orbits? Do all bars extend to their corotation radii? Lastly, various aspects of nested bar systems are only now beginning to be analyzed.

Because the underlying dynamics of bars is strongly nonlinear, their numerical modeling has spearheaded the efforts to understand their orbital structure, ability to channel the angular momentum to the outer disk and

1 Department of Physics and Astronomy, University of Kentucky, Lexington, KY 40506-0055, USA

2 JILA, University of Colorado, Boulder, CO 80309, USA

3 National Institute of Standards and Technology, Boulder, CO 80305-3328, USA

4 Department of Physics, Georgia Southern University, Statesboro, GA 30460, USA dark matter (DM) halo, efficiency in triggering the radial gas inflows, and more. While we have gained some insight to all of these processes, much remains to be done and one expects additional effects to surface.

In this work we aim at deeper understanding of angular momentum redistribution in the disk-halo system facilitated by the stellar bars in the presence of the gas component. Here, we analyze a set of equilibrium collisionless models which differ by a single parameter from each other. In the associated paper, we add the gas component to the system without affecting the mass distribution there (Villa-Vargas et al., Paper II, in preparation). Our collisionless modeling is in a way complementary to that of Athanassoula (2003). We reproduce some of the correlations between the bar, disk and halo properties discussed in the above work and quantify additional correlations found. All these will be compared to models with gas.

Importance of a galactic spheroidal component in bar evolution has been gradually understood, from its supposedly stabilizing effect on the disk (Ostriker \& Peebles 1973; Efstathiou et al. 1982), to serving as disk angular momentum sink (e.g., Sellwood 1980; Weinberg 1985; Debattista \& Sellwood 2000; Athanassoula 2002), and appears to be full of controversies (e.g., Christodoulou, Shlosman \& Tohline 1995a,b; Athanassoula 2008). Study 
of the dominant role of resonance interactions between the bar and surrounding orbits was pioneered by LyndenBell \& Kalnajs (1972) and applied to bar and halo orbits by Tremaine \& Weinberg (1984), Athanassoula (2002), Martinez-Valpuesta, Shlosman \& Heller (2006), Ceverino \& Klypin (2007), Weinberg \& Katz (2007a,b) and Dubinski, Berentzen \& Shlosman (2009). Toomre (1981) argued that the bar growth can be damped by the introduction of an inner Lindblad resonance (ILR), by cutting off the swing amplification mechanism for the $m=2$ modes. Hotter stellar disks are expected to be more stable as well (e.g., Athanassoula \& Sellwood 1986). Counter-intuitively, in the long run, a disk embedded in a more centrally concentrated halo develops a stronger and longer bar (Athanassoula \& Misiriotis 2002).

A comprehensive analysis by Athanassoula (2003) has shown that the efficiency of resonances in angular momentum transfer depends on the DM and stellar dispersion velocities and the DM densities in the vicinity of the major resonances. As the resonances sweep across the phase-space, about $20 \%-30 \%$ of DM particles are locked in the lower order resonances at any given time (Dubinski et al. 2009). Additional aspects of the bar evolution are known to be influenced by the spheroidal components, such as the vertical buckling instability in the bar (Berentzen et al. 2007).

In general, $J$ transfer in the disk-halo systems can depend on three parameters, namely, the particle population near low level resonances and the velocity dispersions in the disk and halo (e.g., Athanassoula 2003). In principle, this means dependence on the halo central mass concentration and on radial velocity dispersions in the disk and the halo. We, therefore, focus on how parameters of the DM halo distribution, such as its mass, concentration, extent, and rms velocities affect the diskhalo evolution and the angular momentum transfer mediated by the stellar bar. Specifically, we are interested in how the presence of gas in the disk influence the angular momentum redistribution in the system. For the purpose of clarity, we separate our discussion of collisionless models (this Paper I) from models with gas (Paper II). Collisionless models are used in order to verify the basic details of bar evolution in order to serve as benchmarks for models with gas. They also are used to calibrate our results to those of Athanassoula (2003).

Our paper is structured as follows: $\S 2$ describes the numerics, initial conditions and model parameters. $§ 3$ provides results on basic evolution of stellar bars in our sets of models and analyzes the redistribution of angular momentum in these systems, while $\S 4$ is focused on correlations between various parameters in the bar-disk-halo models.

\section{NUMERICS AND MODELING}

We use the $N$-body part of the FTM-4.4 hybrid code (e.g., Heller \& Shlosman 1994; Heller, Shlosman \& Athanassoula 2007b) to evolve the stellar disks and DM halos. The gravitational forces are calculated using the FalcON routine (Dehnen 2002) which scales as $O(N)$. The units of mass and distance are taken as $10^{11} \mathrm{M}_{\odot}$ and $10 \mathrm{kpc}$ respectively. This makes the unit of time as $4.7 \times 10^{7}$ yr when $G=1$, and the velocity unit $208 \mathrm{~km} \mathrm{~s}^{-1}$. The gravitational softening is $\epsilon_{\text {grav }}=0.016$ for stars and DM particles. The models consist of a stel- lar disk with $N_{\mathrm{d}}=2 \times 10^{5}$ and of DM halo with $N_{\mathrm{h}}=10^{6}$ particles.

\subsection{Initial conditions}

The initial conditions were created following the prescriptions and density profiles from Hernquist (1990). The mass volume density distribution in the disk is given in cylindrical coordinates by

$$
\rho_{\mathrm{d}}(R, z)=\frac{M_{\mathrm{d}}}{4 \pi h^{2} z_{0}} \exp (-R / h) \operatorname{sech}^{2}\left(\frac{z}{z_{0}}\right)
$$

where $M_{\mathrm{d}}$ is the disk mass, $h$ is a radial scale length and $z_{0}$ is a vertical scaleheight.

The density of the spherical halo is given by

$$
\rho_{\mathrm{h}}(r)=\frac{M_{\mathrm{h}}}{2 \pi^{3 / 2}} \frac{\alpha}{r_{\mathrm{c}}} \frac{\exp \left(-r^{2} / r_{c}^{2}\right)}{r^{2}+\gamma^{2}},
$$

where $M_{\mathrm{h}}$ is the mass of the halo, $r_{\mathrm{c}}$ is a Gaussian cutoff radius and $\gamma$ is the core radius. $\alpha$ is the normalization constant defined by

$$
\alpha=\left\{1-\sqrt{\pi} q \exp \left(q^{2}\right)[1-\operatorname{erf}(q)]\right\}^{-1}
$$

with $q=\gamma / r_{\mathrm{c}}$.

The particle velocities, dispersion velocities and asymmetric drift corrections have been calculated using moments of the collisionless Boltzmann equation. Since models thus constructed are not in exact virial equilibrium, the halo component was relaxed for $t \sim 40$ in the frozen disk potential.

\subsection{Model parameters}

Our goal is to investigate the effect of the DM mass distribution on the evolution of stellar bars in a live diskhalo system. We choose a specific model, defined here as the standard model (SD), where the DM density profiles are specified by three parameters, $M_{\mathrm{h}}, \gamma$ and $r_{\mathrm{c}}$, corresponding to the DM halo mass, DM core radius and the DM Gaussian cutoff radius. Based on the SD model, three sequences of models have been created, each sequence resulting from varying one of the parameters only. In the fourth sequence, we simultaneously modify two parameters at the time, $\gamma$ and $r_{\mathrm{c}}$, in attempt to target the outer halo only, while trying to keep the inner halo unchanged. In the SD model, the halo-to-disk mass ratio in the inner $R=0.6$ is kept to unity. Table 1 lists the values of the SD parameters.

TABLE 1

PARAMETERS OF THE STANDARD MODEL

\begin{tabular}{lr||lr}
\hline \multicolumn{2}{c||}{ HALO } & \multicolumn{2}{c}{ DISK } \\
Parameter & Value & Parameter & Value \\
\hline$N_{\mathrm{DM}}$ & $10^{6}$ & $N_{*}$ & $2 \times 10^{5}$ \\
$M_{\mathrm{h}}$ & 3.15 & $M_{\mathrm{d}}$ & 0.63 \\
$r_{\mathrm{t}}$ & 8.55 & $R_{\mathrm{t}}$ & 1.71 \\
$\gamma$ & 0.1425 & $h$ & 0.285 \\
$r_{\mathrm{c}}$ & 2.85 & $z_{0}$ & 0.057 \\
& & $Q$ & 1.5 \\
\hline
\end{tabular}

Note. $-\overline{Q \text { is the Toomre parameter fixed at } R}=2.4 h$, where $h$ is the thickness of the disk; $r_{\mathrm{t}}$ and $R_{\mathrm{t}}$ are numerical truncation radii in the halo and the disk. All values are given in dimensionless units, $\S 2$. 
The initial mass volume density profile in the disk is kept unchanged in all models. The spatial distribution of the stellar particles is thus identical in all realizations of the initial conditions. On the other hand, the velocities of the stellar particles have been adjusted in each models to provide for the changing rotational support against the combined gravitational potentials of the varying diskhalo systems. The four model sequences are as following:

$M_{\mathrm{h}}$ sequence: the mass of the halo, $M_{\mathrm{h}}$, has been reduced to $70 \%$ and $40 \%$ (models M70 and M40 respectively) of the SD model.

$\gamma$ sequence: the DM core radius $\gamma$ was increased by a factor of 2 and 4 with respect to the SD model (models C30 and C57 respectively).

$r_{\mathrm{c}}$ sequence: the DM Gaussian cuttoff radius $r_{\mathrm{c}}$ was decreased to $77 \%$ and $56 \%$ of that in the SD model (models T22 and T16 respectively).

$M_{\mathrm{h}}-r_{\mathrm{c}}$ sequence: in these hybrid models, MT1 and MT2, both $r_{\mathrm{c}}$ and $M_{\mathrm{h}}$ have been varied, to keep the inner $r<0.1 \mathrm{DM}$ density profile close to that of the SD model.

TABLE 2

Halo Parameters For the MOdel SEQUences

\begin{tabular}{lrrr}
\hline Model & $m_{\mathrm{h}}$ & $\gamma$ & $r_{\mathrm{c}}$ \\
\hline SD & 3.15 & 0.1425 & 2.85 \\
& & & \\
M70 & 2.20 & 0.1425 & 2.85 \\
M40 & 1.26 & 0.1425 & 2.85 \\
& & & \\
C30 & 3.15 & 0.30 & 2.85 \\
C57 & 3.15 & 0.57 & 2.85 \\
& & & \\
T22 & 3.15 & 0.1425 & 2.20 \\
T16 & 3.15 & 0.1425 & 1.60 \\
& & & \\
MT1 & 2.40 & 0.1425 & 2.20 \\
MT2 & 1.71 & 0.1425 & 1.60 \\
\hline
\end{tabular}

Note. - All values are given in dimensionless units, $\S 2$

The DM halo parameters in each model are listed in Table 2. The number of particles and the numerical truncation radii are the same as in the SD model. The halo mass $M_{\mathrm{h}}$ is conserved in sequences $\gamma$ and $r_{\mathrm{c}}$. The DM density profiles of all models after relaxation in the frozen disk potential are shown in Figure 1. The resulting DM density profiles exhibit monotonic decrease along the $M_{\mathrm{h}}$ sequence, show a progressively larger core in the $\gamma$ sequence, move the outer halo mass inward to the inner halo, across $r \sim 1.4$, in the $r_{\mathrm{c}}$ sequence, and increase the outer halo mass while leaving the core unchanged in the hybrid models sequence. The initial circular velocity curves, showing the contributions from the disk, the halo and the total, are displayed in Figure 2, Models M40, C30 and C57 host maximal disks which dominate the potential of the inner part. Models SD, M70, MT1 and MT2 have equal contributions of disk and halo at $t=0$, while T22 and T16 are halo dominated. With the development of the stellar bar, the disk typically becomes even more dominating, although the inner halo is

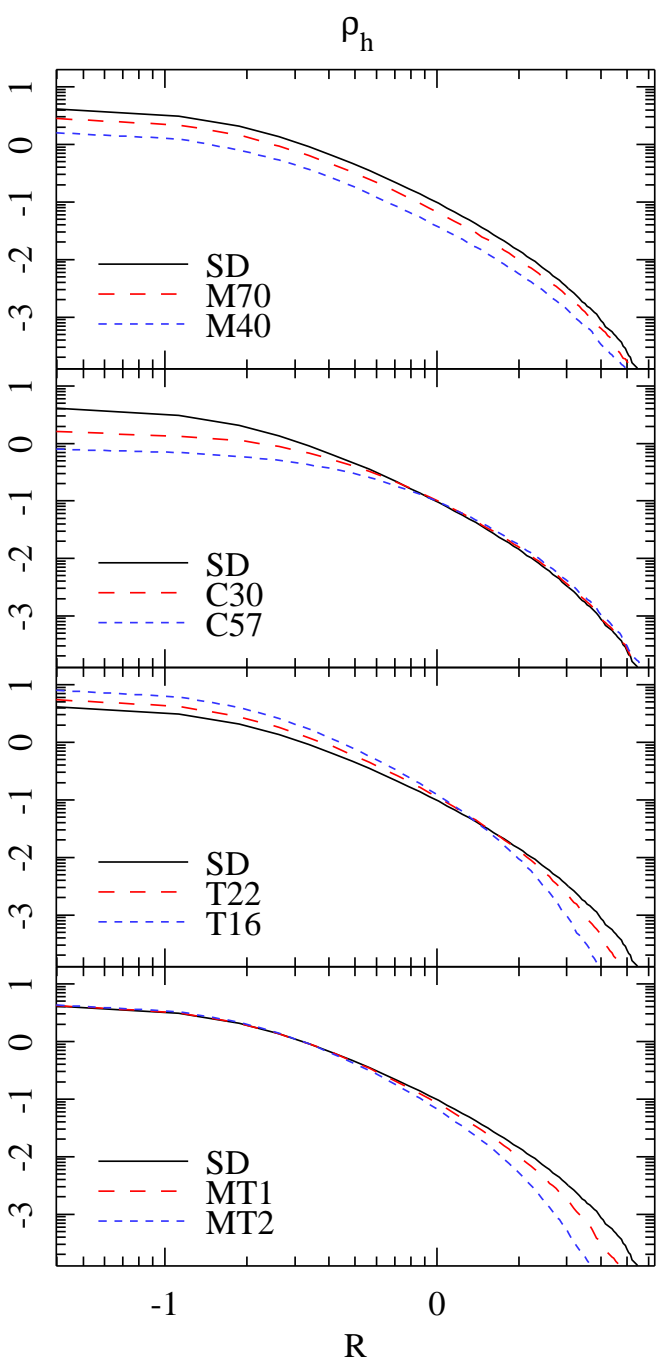

FIG. 1. - The DM halo density profiles at $t=0$ after relaxation in the frozen disk potential. Each panel shows the model sequences. From top to bottom: $M_{\mathrm{h}}, \gamma, r_{\mathrm{c}}$ and the hybrid $M_{\mathrm{h}}-r_{\mathrm{c}}$ sequences.

also dragged in an adiabatic contraction (e.g., Dubinski et al. 2009).

\section{RESULTS}

The model sequences described in Table 2 differ only by the parameters of DM distribution as given by Eq. 2 . These include the total mass of the halo, its core size and the Gaussian truncation radii. As the bar properties and angular momentum evolution are both heavily dependent on the mass distribution in the system, the choice of the free parameters allows us to fine-tune the changes. Because the DM appears to be on the receptive side of the angular momentum transfer, we target the halo as a whole, and its inner and outer parts separately. Discussion and comparison with published models is made in $\S 4$.

\subsection{Bar strength and pattern speed evolution}

To gauge the strength of the bar we use the Fourier amplitude $A_{2}$ of the $m=2$ mode normalized by the $m=0$ mode. It is obtained by various methods - here we show 


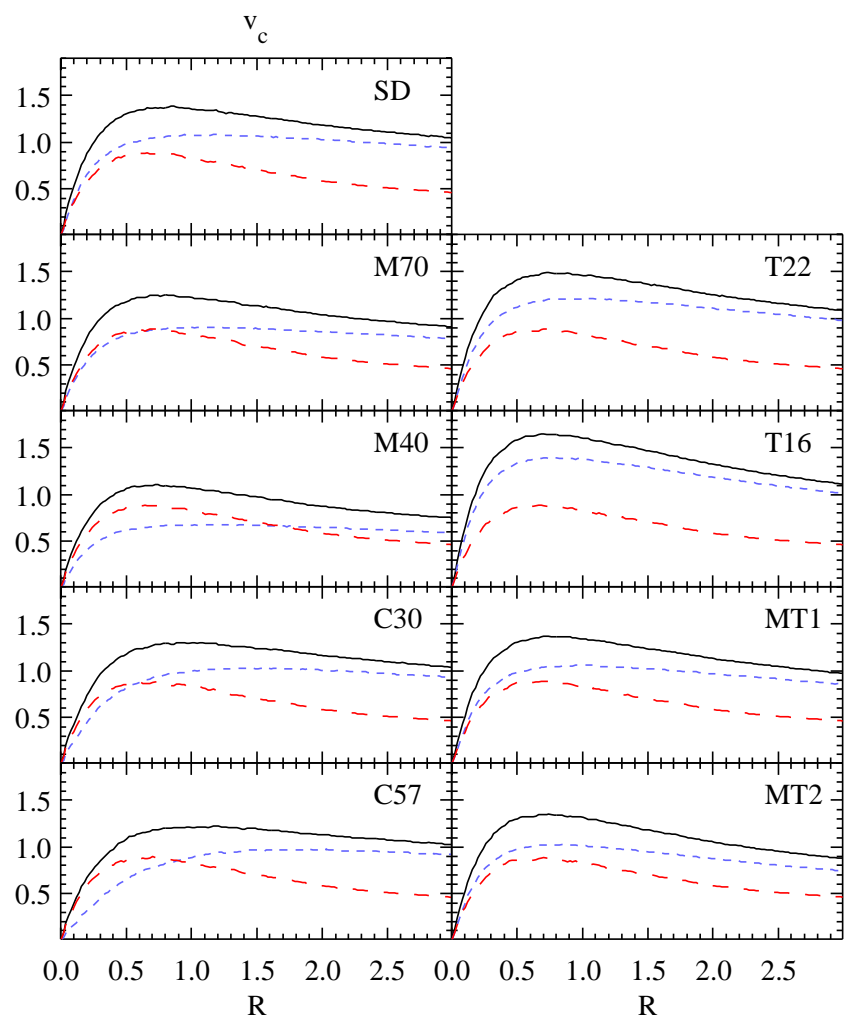

FIG. 2.- Circular velocities of all models at $t=0$ after halo relaxation in the frozen disk potential. Each panel displays the stellar disk rotation curve (red, long dashed), halo (blue, dashed) and the total curve (black, solid).

the results of integration over all the disk, and integration over restricted cylindrical volumes of a particular interest. We define $A_{2}$ in the integration limit over the fixed radial range $R=0.1-1.8$. Second, we define $A_{2 \mathrm{~b}}$ when integrate over $R=0.1-R_{\mathrm{b}}$ range, where $R_{\mathrm{b}}$ is the bar size defined in $\S 3.2$. Intuitively, the second definition reflects the bar properties more fully because $A_{2 \mathrm{~b}}$ is not diluted by the disk properties. Both $A_{2}$ and $A_{2 \mathrm{~b}}$ show some similarities, e.g., the same peaks, raises and drops, and differences, e.g., the relative strength of the peaks (Fig. 3. first and second columns).

Most models show common stages in the evolution of $A_{2}$ and $A_{2 \mathrm{~b}}$ : (1) an initial exponential steep rise, (2) a subsequent peak or plateau followed by a sudden drop, ensued by (3) a more gradual and sustained rise, ending by (4) a saturation of the bar strength. The duration of each stage varies from model to model, but stages "1" and " 2 " are much shorter than stage " 3 ". We refer to stages " 1 " and "2" as the dynamical evolution of the bar, and stages " 3 " and " 4 " as the secular evolution of the bar. Models M40 and T16 are the exceptions. The former model has the least massive halo and exhibits a prolonged secular growth of the bar after the first peak. The bar amplitude does not reach saturation even in the Hubble time. The latter model has the most massive halo core and the bar strength never shows a clear first peak seen in all other models. However a careful analysis of $A_{2}$ and especially $A_{2 \mathrm{~b}}$ reveals that the exponential growth is terminated at $t \sim 120$ and the subsequent evolution lucks the vertical buckling instability (see $\S 3.4$ ), but oth- erwise follows the path of other models. We take a closer look at these trends in $\S 4$. We note that our decision to create one-parameter sequences naturally leads to a wide spectrum of bar properties and can include some of the extreme behavior.

Some clear trends of the bar strength behavior can be observed along each of the sequences defined in $§ 2.2$. First, in all models, except the two exclusions mentioned above, $A_{2}$ and $A_{2 \mathrm{~b}}$ have saturated by $t \sim 230$. Because $A_{2 \mathrm{~b}}$ is tailored for the bar, the saturation of this parameter is more obvious (more about this in \$3.3). Second, making the inner halo less massive (i.e., less centrally concentrated), either by reducing the total halo mass or by increasing the size of the central core (sequences $M_{\mathrm{h}}$ and $\gamma$, respectively), results in a shorter rise time of the bar instability and hence brings up the bar earlier. Third, increase in the mass of the inner halo and decrease in the outer one, has the most dramatic effect on the bar strength, substantially increasing the timescale of the bar instability. This hints at DM mass concentration being important rather than the total halo mass. Varying the mass of the outer halo alone has a much smaller effect on the bar. Finally, in all one-parameter sequences, the first $A_{2}$ or $A_{2 \mathrm{~b}}$ peak forms a progressively more extended plateau for less concentrated halos, respectively. Again T16 is an exclusion.

Furthermore, $A_{2}$ and $A_{2 \mathrm{~b}}$ show high frequency variability in some models (e.g., Figure 4), being strongest in models SD, C30 and T22. This variability can also be seen in $R_{\mathrm{b}}$ and $\Omega_{\mathrm{b}}$ evolution, the bar length and its pattern speed. This variability is limited to the time of the bar secular growth and dies out with saturation of the bar strength, $A_{2}$ and $A_{2 \mathrm{~b}}$. These oscillations coincide in time with the presence of four short arms located close to the bar end, and two spirals in the outer disk beyond the bar radius. Both sets of spirals have pattern speeds larger than that of the bar. The presence of more than one strong pattern of waves or modes in the disk can be the result of a non-linear mode coupling (e.g., Tagger et al. 1987; Sygnet et al. 1988) that gives rise to beat waves of a frequency $\omega_{\text {beat }}=\omega_{1} \pm \omega_{2}$ and the azimuthal wave number $m_{\text {beat }}=m_{1} \pm m_{2}$, where $m_{1}$ and $m_{2}$ are the (azimuthal) wave numbers of the interacting waves and $\omega_{1}$ and $\omega_{2}$ are their frequencies, $\omega=m \Omega$, where $\Omega$ 's are their pattern speeds. This type of coupling has been observed and quantified in numerical simulations (e.g., Masset \& Tagger 1997; Martinez-Valpuesta 2006). Debattista \& Sellwood (2000) has also observed the high frequency variability, but no quantitative analysis of the mode coupling was attempted.

We test whether the bar and the outer two spirals, both $m=2$ modes, couple to give rise to the observed beat mode $m_{\text {beat }}=4$ arms. We verify that the frequency $\omega_{\text {beat }}$ of the $m_{\text {beat }}=4$ mode is actually the sum of the frequencies of the interacting modes. Table 3 shows the frequencies $\omega_{\mathrm{b}}=2 \Omega_{\mathrm{b}}, \omega_{\mathrm{s}}=2 \Omega_{\mathrm{s}}$ and $\omega_{\text {beat }}$ obtained with the mode coupling analysis, with an error of \pm 0.02 . Here $\Omega_{\mathrm{s}}$ is the pattern speed of the outer $m=2$ spirals. The measured frequency of the beat mode (column 5) matches the frequency predicted by the non-linear coupling (column 6). We can explain the oscillations in $A_{2}$ as resulting from superposition of the major axis of the bar with the outer spirals, which are the second strongest mode (after the bar itself) present in the disk. The fre- 

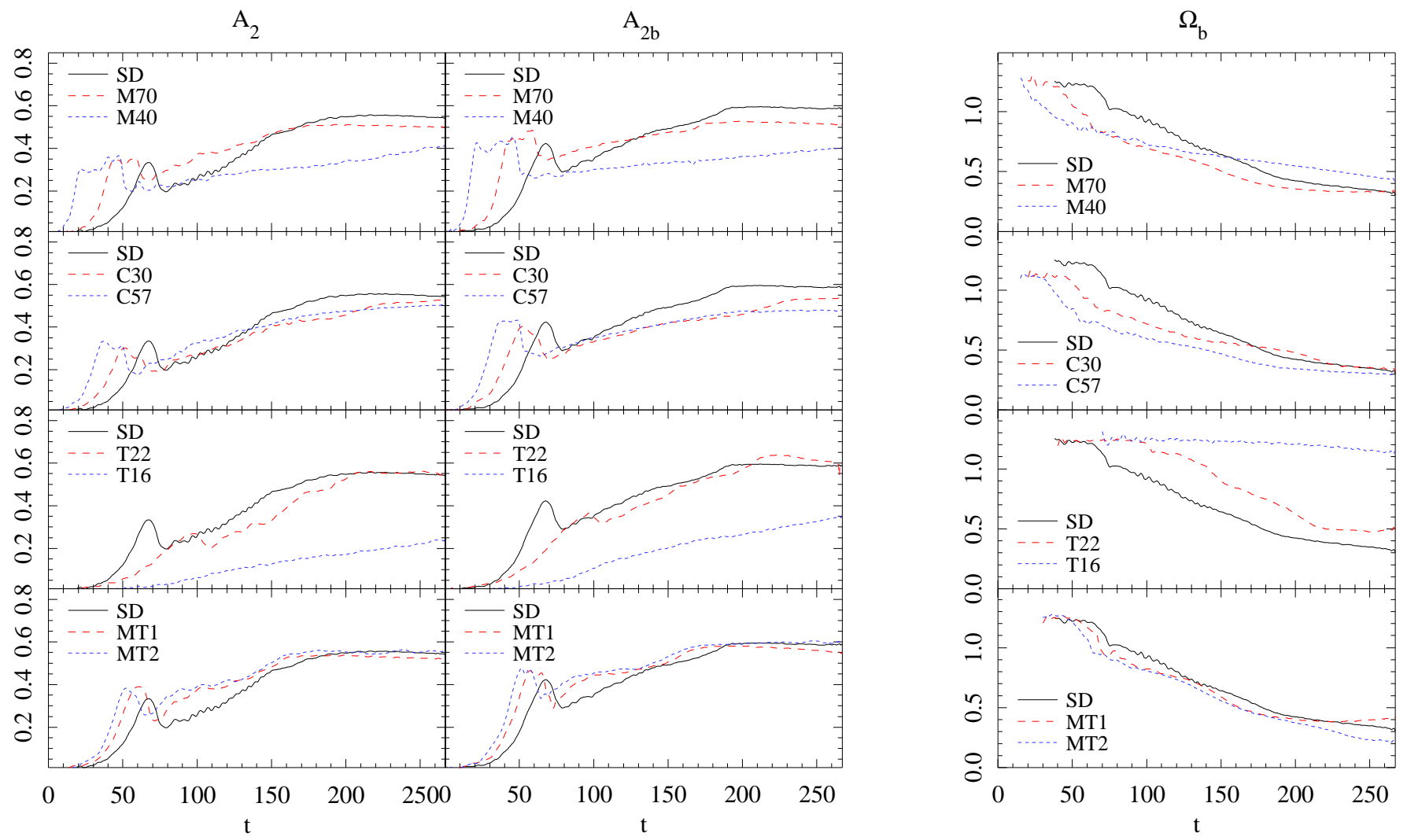

Fig. 3. - Time evolution of normalized bar amplitudes, $A_{2}$ (left column) and $A_{2 \mathrm{~b}}$ (middle column), and the bar pattern speed $\Omega_{\mathrm{b}}$ (right column) in all models. The model sequences are indicated in the upper left corners. All the data has been smoothed with a high frequency Fourier filter.

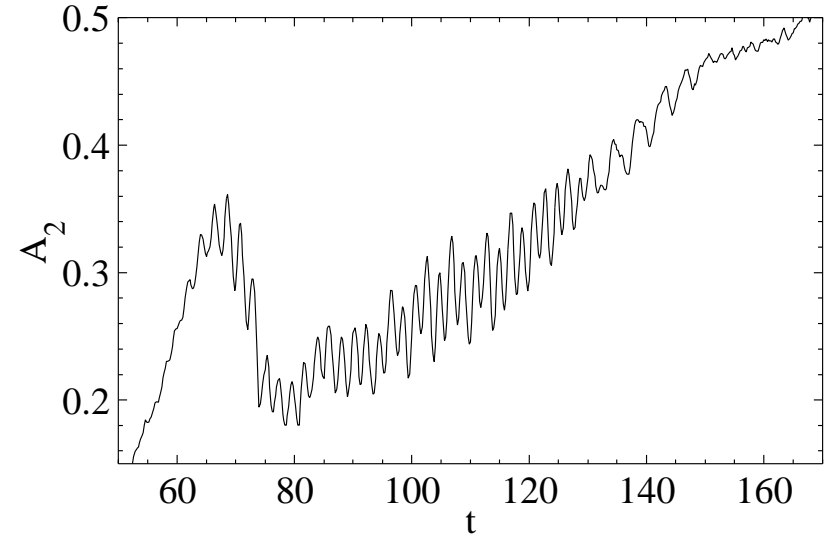

FIG. 4.- Close up of the $A_{2}$ evolution in the SD model, showing the high frequency oscillations during $t \sim 70-160$. No smoothing has been applied to the data.

quency $f_{\mathrm{A} 2}$ of the $A_{2}$ oscillations must thus be equal to the frequency at which the spirals align with the bar $f_{\mathrm{sb}} \equiv\left(\omega_{\mathrm{s}}-\omega_{\mathrm{b}}\right) / 2 \pi$. As can be seen from columns 7 and 8 , these two frequencies coincide within the error limits.

Figure 3 shows the evolution of $\Omega_{\mathrm{b}}$ in our models, which is well defined by the time $A_{2} \sim 0.05$. In all models, the initial value of $\Omega_{\mathrm{b}}$ is very similar, with the scatter of \pm 0.1 . Although the details of the evolution of
TABLE 3

MODE COUPLING IN THE DISK

\begin{tabular}{lrrrrrrr}
\hline Model & Time & $\omega_{\mathrm{b}}$ & $\omega_{\mathrm{s}}$ & $\omega_{\text {beat }}$ & $\omega_{\mathrm{b}}+\omega_{\mathrm{s}}$ & $f_{\mathrm{A} 2}$ & $f_{\mathrm{sb}}$ \\
\hline SD & 106 & 1.80 & 4.86 & 6.66 & 6.66 & 0.49 & 0.49 \\
$\mathrm{C} 30$ & 113 & 1.32 & 4.24 & 5.58 & 5.56 & 0.45 & 0.46 \\
T22 & 149 & 1.80 & 5.72 & 7.58 & 7.52 & 0.61 & 0.62 \\
\hline
\end{tabular}

Note. - (1) the model; (2) the time at which the measurements were taken; (3) $\omega_{\mathrm{b}}$; (4) $\omega_{\mathrm{s}}$; (5) $\omega_{\text {beat }} ;(6)$ the expected frequency of the beat mode $\omega_{\mathrm{b}}+\omega_{\mathrm{s}} ;(7)$ measured frequency of $A_{2}$ oscillations (8) frequency of alignment of bar and spirals.

$\Omega_{\mathrm{b}}$ differ from model to model, most importantly, often shows an (anti)-correlation with $A_{2}$ and $A_{2 \mathrm{~b}}$, as emphasized already by Athanassoula (2003). This is not always observed in cosmological simulations of disk evolution (Heller, Shlosman \& Athanassosula 2007a; Romano-Diaz et al. 2008). We find, however, that the anti-correlation between the bar strength and its pattern speed are limited to times prior to saturation of $A_{2}$ and $A_{2 \mathrm{~b}}$. In $\S 3.3$ we show that this corresponds to times when the bar corotation radius lies within the disk. In three models, M70, T22 and MT1, the $A_{2}-\Omega_{\mathrm{b}}$ correlation is maintained even after the corotation leaves the disk - in these models the total $J$ of the disk is conserved during this time period of flat $A_{2}$ and $\Omega_{\mathrm{b}}$.

A close examination of $\Omega_{\mathrm{b}}$ evolution in Fig. 3 reveals prolonged periods of $\Omega_{\mathrm{b}} \sim$ const. These are found either in the dynamical phase, prior to the first buckling but 
when the bar is already sufficiently strong (SD, M70, C57), or during the late stage of secular evolution, especially pronounced in M70, T22 and MT1, and to a lesser degree in C57. These latter cases are of most interest to us. A similar behavior was discussed before by Valenzuela \& Klypin (2003), who related it to an abnormally low dynamical friction of the bar against the background. We return to this issue in $\S 3.6$.

\subsection{Evolution of the bar length}

The semi-major axis of the bar, $R_{\mathrm{b}}$, is defined here as the radius where the bar equatorial ellipticity drops by $15 \%$ off its peak. Martinez-Valpuesta et al. (2006) has tested this method by contrasting it with the most reliable way of determining the bar size using the last stable orbit supporting the bar (Martinez-Valpuesta et al. 2006). It seems robust when applied after the first maximum of the bar strength. The ellipticity of the bar at different radii is obtained by fitting ellipses to the isodensity contours in the face-on disk. Figure 5 displays the evolution of $R_{\mathrm{b}}$ for all the models.

Similarly to the evolution of $A_{2}$ and $A_{2 \mathrm{~b}}$, the bar length has initial period of a fast growth that reaches maximum, sometimes followed by a drop and subsequent sustained but slower rise, in most models. Some models show a degree of saturation, toward the end. The first peak in $R_{\mathrm{b}}$ always coincides in time with that of $A_{2}$, except in the model T16 which lacks it. The peak in $R_{\mathrm{b}}$, however, is not as pronounced as that in $A_{2}$ and there are additional caveats. As in $A_{2}$, the initial growth varies with a model in such a way that, in models with the less centrally concentrated halos, the time span of the initial growth is shorter. Furthermore, in some cases, additional drop in the bar length can be seen after some time of the secular growth, in the time range of $t \sim 150-260$. This drop appears to be related to the formation and disappearance of the ansae at each tip of the bar (Martinez-Valpuesta et al. 2006). The ansae eventually 'detach' from the bar and becomes misaligned with its major axis. The transition to this misalignment results in a fast decrease of the bar length, taking place at $t \sim 180$ for model SD, $\sim 190$ for M70, 22 for C30, 190 for C57, 205 for T22, and 165 for MT1 and MT2.

In general, evolution in the bar length does not necessarily go in tandem with the changes in $A_{2}$ and is much less monotonic. For example, the drop in $R_{\mathrm{b}}$ around $t \sim 180$ in the SD model has no counterpart in the $A_{2}$ or $A_{2 \mathrm{~b}}$ evolution. Of course, a stronger $A_{2}$ does not mean a longer bar, as seen in the last $\Delta t \sim 80$ of the evolution of the sequence $M_{\mathrm{h}}$ models.

Overall, the $M_{\mathrm{h}}$ and $r_{\mathrm{c}}$ sequences show a substantial dispersion in the bar sizes at the end of the simulations, while two other sequences end up with very similar bars. In all sequences, the bars differ substantially during the intermediate times, especially after the first buckling time.

\subsection{Evolution of the bar corotation radius}

The bar corotation radius $R_{\mathrm{cr}}$ has been computed using linear approximation (the second column in Fig. 5 ). It grows with time as a consequence of the bar slowdown. $R_{\mathrm{cr}}$ keeps growing throughout the simulation, the exceptions are models M70, T22 and MT1, in which a plateau is reached. We use this characteristic radius in order to construct and follow the evolution of two important ratios, namely, $R_{\mathrm{cr}} / R_{\mathrm{b}}$ and $R_{\mathrm{cr}} / R_{\mathrm{d}}$.

The position of $R_{\text {cr }}$ has a profound effect on the angular momentum transfer from the bar region to the outer disk and the DM halo (Athanassoula 2002; MartinezValpuesta et al. 2006). The DM particles at the corotation resonance are responsible for much of the angular momentum absorption by the halo. Hence, the availability of halo or outer disk resonant particles is of the prime importance to the evolution of the bar. Specifically, it is important to know whether $R_{\mathrm{cr}}$ lies within the stellar disk in our models at all times. We define $R_{\mathrm{d}}$ as the disk radius which encloses $98 \%$ of the disk mass, and the evolution of the ratio $R_{\mathrm{cr}} / R_{\mathrm{d}}$ is shown in Figure 5 , At the time of the bar formation, $R_{\mathrm{cr}} / R_{\mathrm{d}}$ lies between 0.5 and 0.8 in all models. This ratio raises with time enough to move the corotation out of the disk with the exception of models M40 and T16, where bars grow at the slowest pace during secular evolution. The growth of the bar seems to be sensitive to the moment at which $R_{\text {cr }}$ reaches the disk edge - even if the growth continues, it proceeds at a slower pace. This change happens with a slight but measurable delay of $\Delta t \sim 20$. Moreover, as noted before, the detachment of the ansae from the bar happens just before $R_{\mathrm{cr}} / R_{\mathrm{d}} \sim 1$, which manifests itself by a sharp drop in the bar length.

We pay a special attention to the bar evolution when $R_{\mathrm{cr}} / R_{\mathrm{d}} \sim 1$. Fig. 5 displays an interesting behavior of this ratio in the above regime - it flattens with time before resuming its growth. Analyzing this behavior, we find that as the $R_{\text {cr }}$ moves outside the disk, some stellar particles become trapped at $R_{\mathrm{cr}} . \quad m=2$ spiral arms appear connecting the outer edge of the disk to these trapped particles. This is characteristic of all models where the above conditions exist. At $t \sim 200-250$, depending on the model, the spirals dissolve, and their particles form an amorphous cloud outside the disk. We return to this issue in $§ 3.6$.

The bar must lie of course inside its $R_{\mathrm{cr}}$, because orbits outside $R_{\mathrm{cr}}$ do not support the bar. The morphology of the offset (gas) shocks in the numerical bars has been argued to constrain the ratio $R_{\mathrm{cr}} / R_{\mathrm{b}}$, restricting it to $R_{\mathrm{cr}} / R_{\mathrm{b}}=1.2 \pm 0.2$ (Athanassoula 1992; see also early work by van Albada \& Sanders 1982). Limiting our discussion of this ratio only to times when $R_{\mathrm{cr}} / R_{\mathrm{d}} \leq 1$, we observe it dropping to the range $\sim 1.0-1.4$ after the first buckling, and staying generally below $\sim 1.8$ until the $R_{\text {cr }}$ moves out of the disk. Model T16 (no buckling!) does not follow this trend and rather shows a monotonic decay to $\sim 1.9$ at the end of the run. On the other hand, M40 - the only other model with $R_{\text {cr }}$ always in the disk, displays a remarkably constant $R_{\mathrm{cr}} / R_{\mathrm{b}}=1.2 \pm 0.2$ almost over the entire run (Figure 5). Naturally, after $R_{\text {cr }}$ moves out of the disk, this ratio is bumped to above 2 . In Paper II, this ratio will be tested against the shape of the offset shocks in the gas.

\subsection{Vertical buckling in the bar}

The $N$-body bars undergo phases of vertical buckling instability where transient asymmetries develop in the form of vertical oscillations of the equatorial plane and asymmetric thickening of the disk (e.g., Combes \& Sanders 1981; Combes et al. 1990; Pfenniger \& Friedli 1991; Raha et al. 1991), and results in the formation 

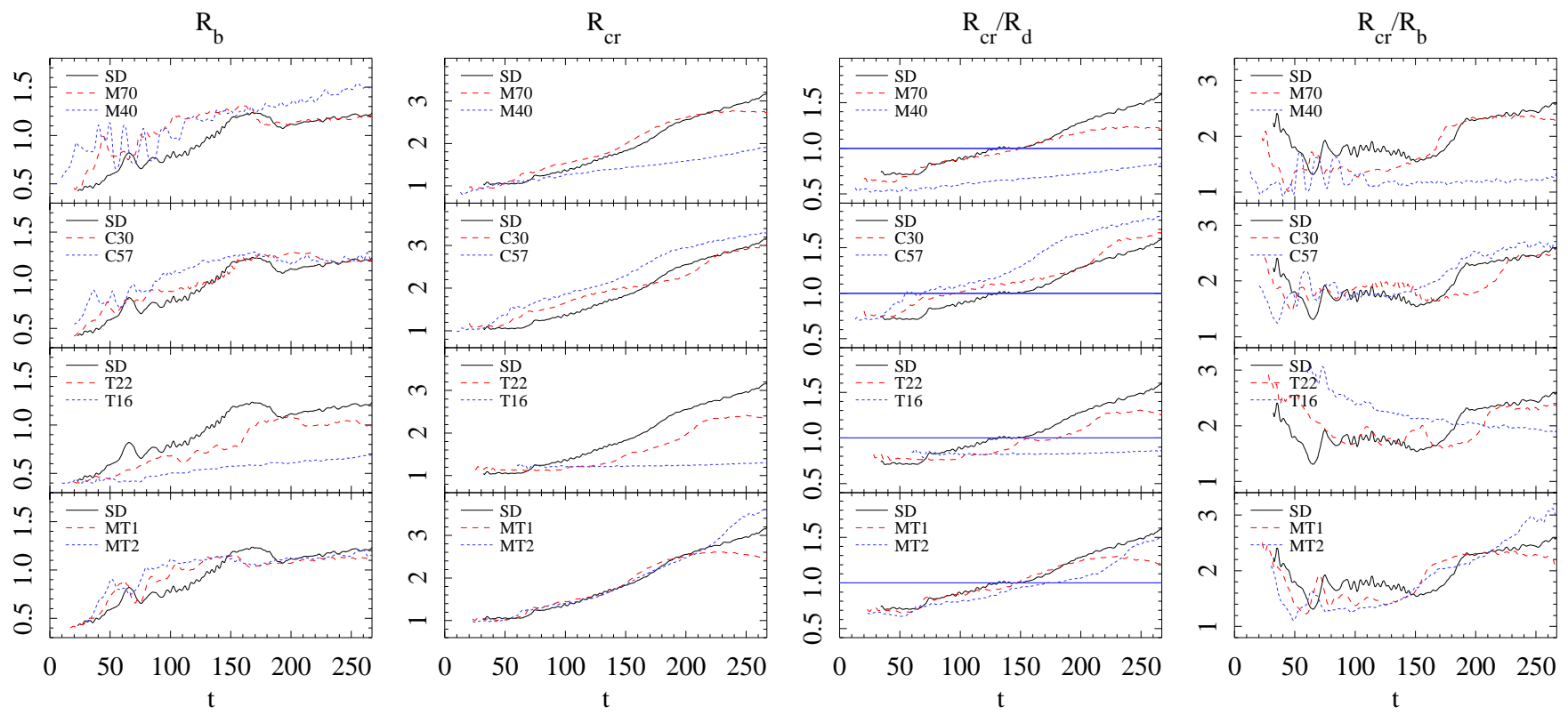

FIG. 5.- Time evolution of (from left to right) $R_{\mathrm{b}}, R_{\mathrm{cr}}, R_{\mathrm{cr}} / R_{\mathrm{d}}$ - ratio of $R_{\mathrm{cr}}$ to the radius of the disk, and $R_{\mathrm{cr}} / R_{\mathrm{b}}$. The $R_{\mathrm{cr}} / R_{\mathrm{d}}=1$ line has been drawn as a reference. SD model (continuous line) is shown in each panel for comparison.

of the so-called peanut/boxy-shaped bulges (Kormendy \& Kennicutt 2004 and refs. therein). These phenomena can be recurrent and affect different parts of the bar (Martinez-Valpuesta et al. 2006; Berentzen et al. 2007). We measure the buckling asymmetry with the Fourier coefficient $A_{1, \mathrm{z}}$ of the $m=1$ component in the $r z$-plane, again normalized by the $\mathrm{m}=0$ mode. In all our models, except T16, we detect at least a single phase of the vertical buckling. The buckling always happens simultaneously with the first $A_{2}$ drop (e.g., Martinez-Valpuesta \& Shlosman 2004), which we confirm here. In all the model sequences, models with less centrally concentrated halo exhibit earlier and stronger buckling. This happens because $A_{2}$ reaches its first maximum earlier in these models. We do not find any clear correlations between the strength and time of the second buckling and the initial properties of the models. In all cases of repeated bucklings, $R_{\text {cr }}$ lies within the disk.

We note that T16 does not exhibit the drop in $A_{2}$ or $A_{2 \mathrm{~b}}$, but does show a break in their slopes around $t \sim 120$. Moreover, while all our models develop peanut/boxy bulges abruptly after these drops, T16 develops this bulge gradually over the secular stage of its evolution. This model appears as a nice example of a secular buildup of peanut/boxy bulges as a result of the diffusive action of the vertical inner Lindblad resonance (Friedli \& Pfenniger 1990; Martinez-Valpuesta \& Shlosman 2004). Berentzen et al. (2007) have shown that mass concentrations resulting from gas accretion to the center damp the vertical buckling and alter the bulge shape, making it more elliptical, if the gas fraction in the disk is high. T16 is a purely collisionless model with the most centrally concentrated halo. It is possible that a qualitatively different behavior is the corollary of this concentration. Clearly, the action of the vertical resonance leads to a secular and not dynamical buildup of a peanut-shaped bulge in this case.
We also note that in all models the nonlinear inner Lindblad resonance (ILR) first appears only after the first buckling, although the linear analysis claims their existence from the start (Pfenniger \& Friedli 1991; MartinezValpuesta et al. 2006). The appearance of this resonance is the consequence of the increase in the central mass concentration, by a factor of $\sim 2$, as a result of the bar buckling, which drags inward the DM as well (Dubinski et al. 2009).

\subsection{Evolution of bar-to-disk mass ratio}

The bar increases its mass by capturing particles from the disk within its $R_{\mathrm{cr}}$. In Figure 7 we plot the fraction of the stellar mass contained inside the bar, $M_{\mathrm{bar}} / M_{\mathrm{d}}$. To estimate the bar, we sum the particle masses within a rectangular box aligned with the bar major axis, with dimensions given by the major and minor axes of the bar and $|z| \leq 0.5$.

By the end of the run, the bar has captured between $75 \%$ and $85 \%$ of the disk mass (the exception is model T16 whose bar growth has been severely delayed by the halo mass concentration). The initial stage of bar formation is associated with an intense capture of disk particles. Eventually this rate declines, and even though the bar mass keeps growing it does so at a much more modest pace. The growth of $M_{\mathrm{bar}} / M_{\mathrm{d}}$ clearly correlates with the evolution of the bar size in Fig. [5 (left frame) an increase/decrease in one of them corresponds with an increase/decrease in the other. Typically, the growth of the bar size and of $M_{\mathrm{bar}} / M_{\mathrm{d}}$ saturates when the $R_{\mathrm{cr}} / R_{\mathrm{d}}$ is driven above unity. However, surprisingly, there are exclusions of this behavior, e.g., C57.

We do not find correlation between the bar mass and $A_{2}$. It may happen that the bar keeps capturing mass while $A_{2}$ is saturated, as in model SD at $t \sim 210$ and later on. In summary, the ratio $M_{\mathrm{bar}} / M_{\mathrm{d}}$ saturates around 0.8 in in all models, except T16. 


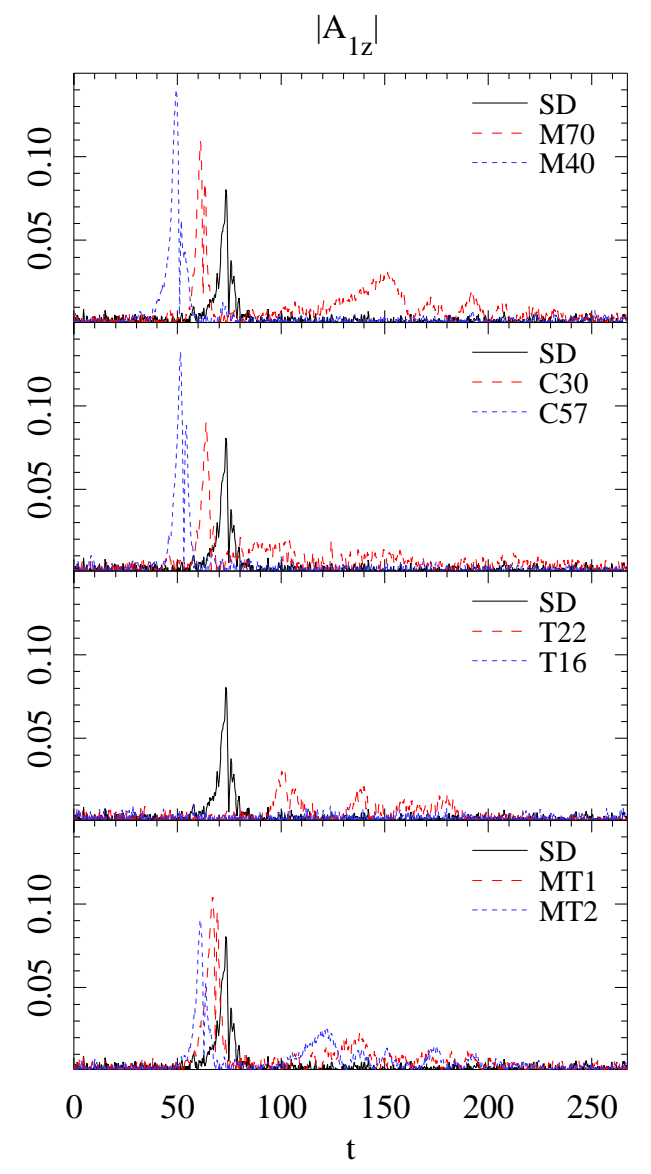

FIG. 6. - Evolution of the bar vertical asymmetry given by the Fourier coefficient $\left|A_{1, z}\right|$ of the $m=1$ mode in the $r z$-plane corotating with the bar major axis. No filtering was applied to this data.

\subsection{Evolution of the angular momentum}

The backbone of the bar is composed from material moving along eccentric orbits of relatively low angular momentum, compared to the circular orbits of the same energy. Moreover growing bars consume more circular orbits, i.e., those with larger $J / E$ ratio, redistributing their angular momentum to the outer disk and halo. The capacity of different regions of the disk and halo to emit and absorb angular momentum is thus of a prime importance to the bar evolution, and largely determine its ability to grow by trapping additional disk orbits. Rather than estimate the effect of the resonances on this process (Athanassoula 2002; Martinez-Valpuesta et al. 2006), we focus on the total $J$ transfer within the disk and to the halo, i.e., accounting for the resonant and non-resonant $J$ redistribution. In Figure 8 we plot the evolution of the $z$ component of the total angular momentum of the disk, $J_{\mathrm{d}}$, as well as in the cylindrical volumes centered on the symmetry axis of the disk and separated by $R_{\mathrm{cr}}$, i.e., $R \leq R_{\mathrm{cr}}\left(J_{\mathrm{d}, \text { in }}\right)$ and $R_{\mathrm{cr}} \leq R$ ( $\left.J_{\mathrm{d} \text {,out }}\right)$, and similarly for the halo (Fig. 91). Since the total angular momentum in each model is conserved, the evolution of $J_{\mathrm{h}}$ in the halo is an inverted mirror image of that in the disk.

The initial angular momentum, $J_{\mathrm{d}}(t=0)$, of the disk

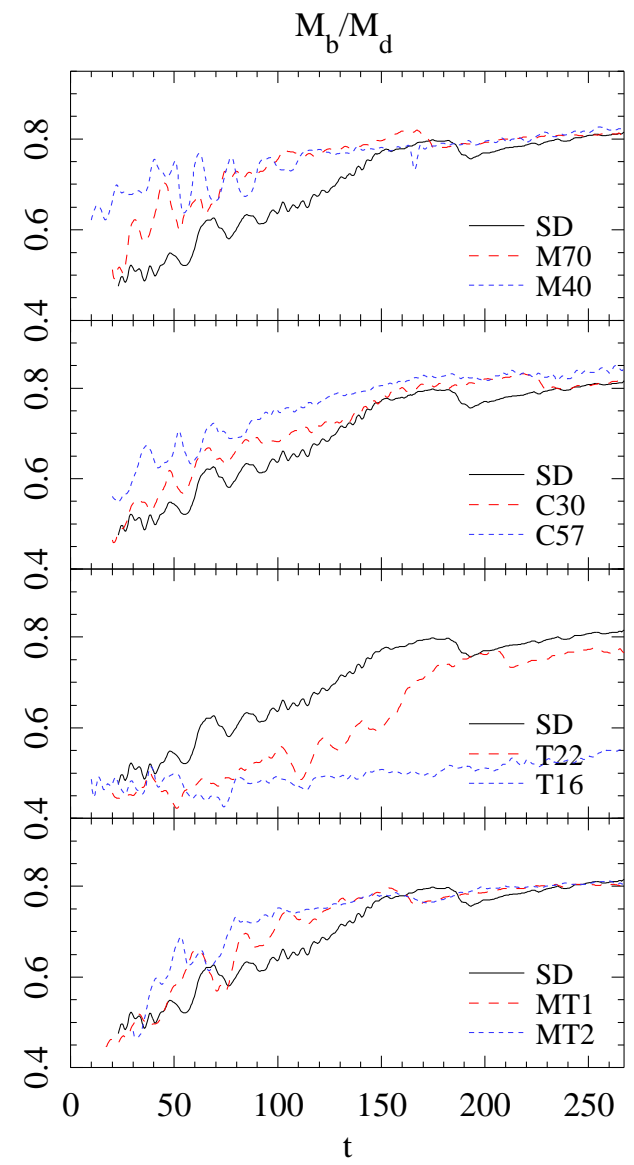

FiG. 7.- Evolution of bar-to-disk mass ratio $M_{\mathrm{bar}} / M_{\mathrm{d}}$.

varies from model to model due to the differences in the halo mass distribution. It changes very little before the vertical buckling in the bar, while $J_{\mathrm{d} \text {,in }}$ and $J_{\mathrm{d} \text {,out }}$ anticorrelate. This means that the halo gains a negligible amount of $J$, which flows nearly exclusively to the outer disk during this phase (see also Fig. 9). After the buckling, $J_{\mathrm{d}}$ and $J_{\mathrm{d} \text {,out }}$ drop monotonically till the end of the

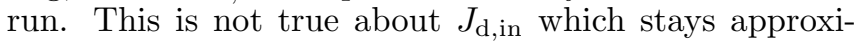
mately constant until the time when $R_{\text {cr }}$ leaves the disk (Fig. 8. see also Martinez-Valpuesta et al. 2006). How this is possible?

While it is not surprising that the loss of $J$ within the corotation leads to an outward motion of $R_{\mathrm{cr}}$, the apparent 'conspiracy' in $J_{\mathrm{d} \text {,in }} \sim$ const. over such an extended time period is somewhat puzzling. It hints at some regulation mechanism which fine tunes the loss of angular momentum to the outer disk and to the halo by gravitational torques with its influx across the corotation. We attempt to answer this question, using obvious simplifications: the explanation of this phenomenon lies in the intricacies of $J_{\mathrm{d} \text {,in }}$ balance and the resulting behavior of $\Omega_{\mathrm{b}}$. The angular momentum within $R_{\mathrm{cr}}$ is

$$
J_{\mathrm{d}, \text { in }}=2 \pi \int_{0}^{R_{\mathrm{cr}}(t)} d R R^{2} \Sigma(R, t) \bar{v}_{\mathrm{t}},
$$

where $\bar{v}_{\mathrm{t}} \equiv \alpha v_{\mathrm{c}}$ is the average tangential velocity of par- 

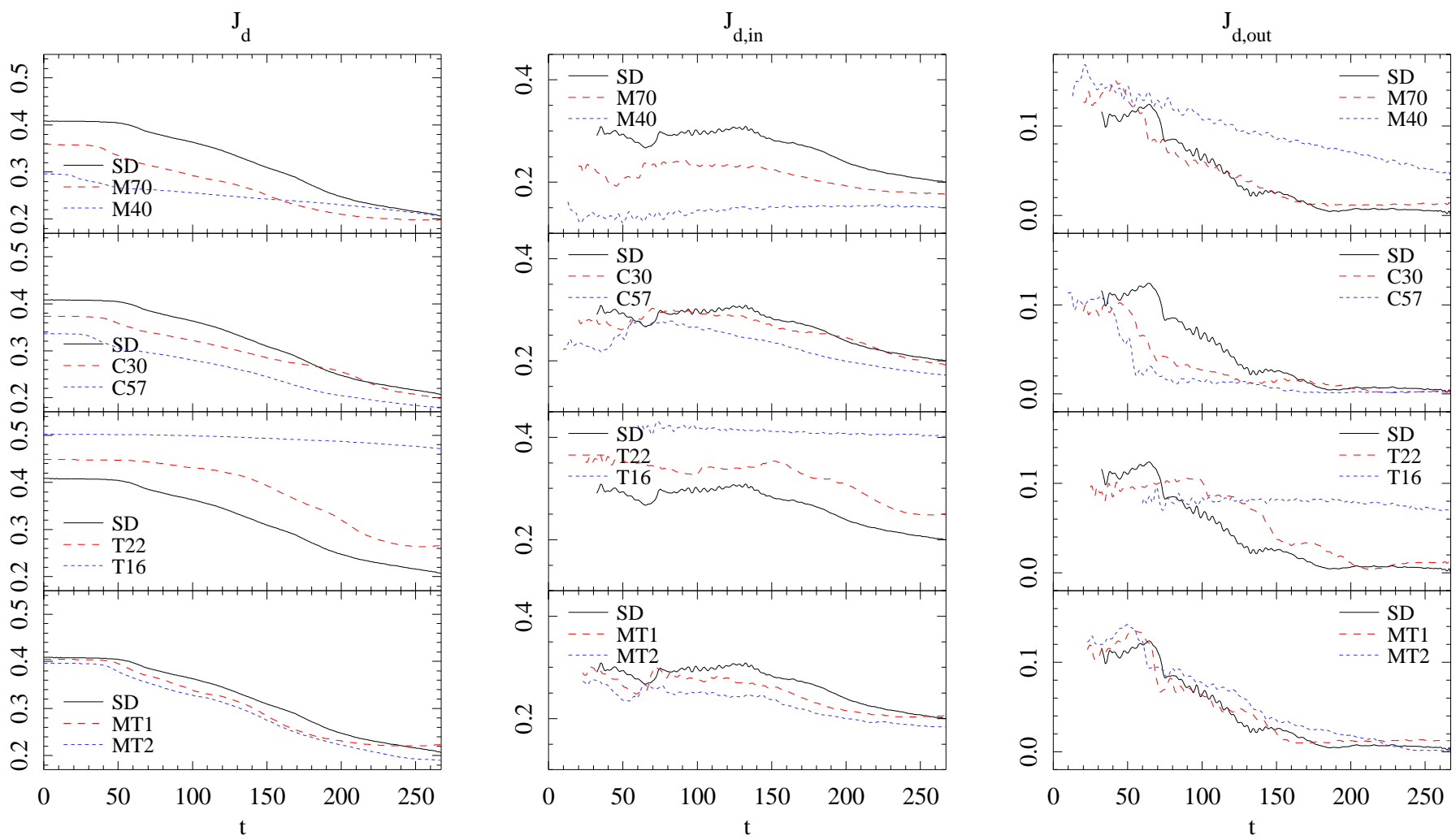

FIG. 8. - Evolution of the angular momentum, $J_{\mathrm{d}}$, in the disk enclosed by the cylindrical volumes: $R \leq R_{\mathrm{d}}$ (left), by $R \leq R_{\mathrm{cr}}$ (center), $R_{\mathrm{cr}} \leq R \leq R_{\mathrm{d}}$ (right). The vertical extent of integration is taken as $z= \pm 0.3$. Note that the vertical scales are different in each column.

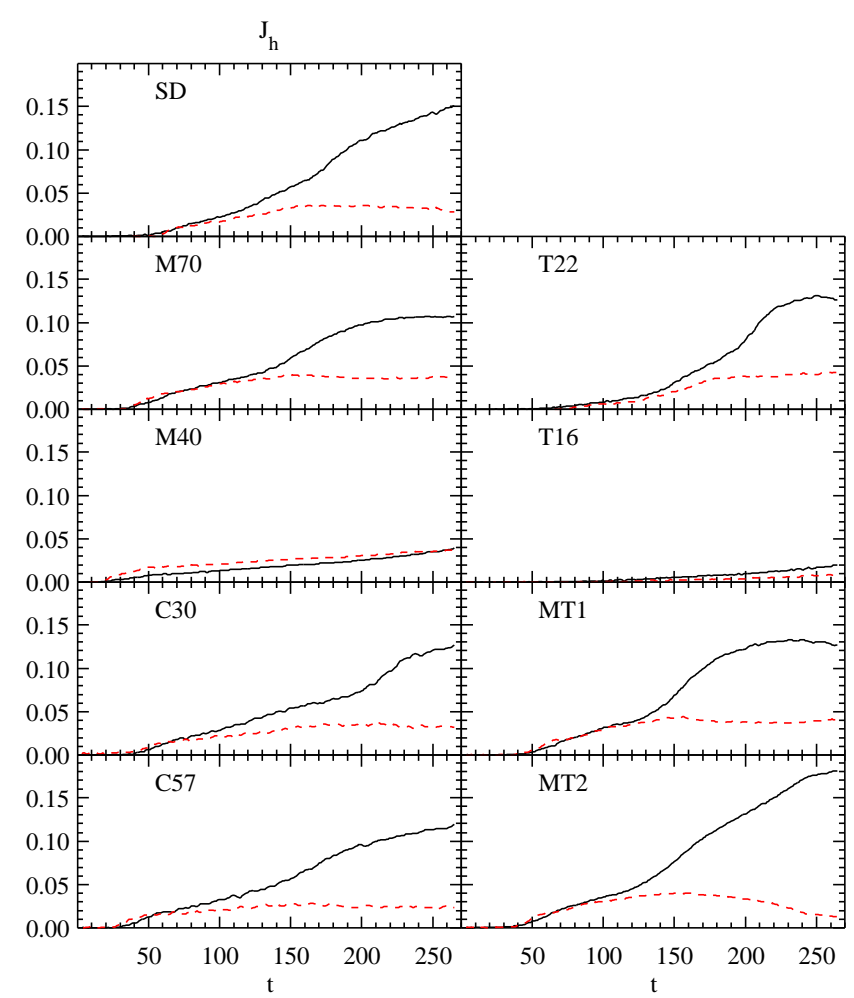

FIG. 9.- Evolution of the $z$ components of the halo angular momenta, $J_{\mathrm{h}, \text { in }}$ (solid, black) and $J_{\mathrm{h}, \text { out }}$ (dashed, green), enclosed by the cylindrical volumes $R \leq R_{\mathrm{cr}}$ and $R \geq R_{\mathrm{cr}}$, and $z= \pm 2$. ticles inside $R_{\mathrm{cr}}$, which also defines the coefficient $\alpha$. We require $J_{\mathrm{d}, \text { in }} \sim$ const. between the times of buckling ${ }^{5}$ and when $R_{\mathrm{cr}} / R_{\mathrm{d}} \sim 1$, and take the time derivative of $J_{\mathrm{d} \text {,in }}$ using the Leibniz formula,

$$
\begin{aligned}
\frac{1}{2 \pi} \frac{d J_{\mathrm{d}, \text { in }}}{d t}= & \int_{0}^{R_{\mathrm{cr}}} R^{2} \frac{d}{d t}\left[\Sigma(R, t) \bar{v}_{\mathrm{t}}\right] d R+ \\
& +v_{\mathrm{c}} R_{\mathrm{cr}}^{2} \Sigma\left(R_{\mathrm{cr}}, t\right) \frac{d R_{\mathrm{cr}}}{d t}=0,
\end{aligned}
$$

where $\Sigma$ is the disk surface density which can be obtained from eq. 1 by integrating over $z$. We assume $v_{\mathrm{c}} \sim$ const. This is justified because contribution of $J$ within the velocity turnover radius is small and at larger radii, $v_{\mathrm{c}}$ is dominated by the isothermal halo and is nearly independent of radius (Fig. 2). The first term in eq. 5 describes the emission of $J$ by the inner disk, within an instantaneous $R_{\text {cr }}$, due to the torque $T$ imposed by the DM and the outer disk. For brevity, we assume that the stellar bar extends to $R_{\mathrm{cr}}$ and this torque acts upon it. In other words, all the material within $R_{\mathrm{cr}}$ resides in the bar. The second term in eq. 5 represents the influx of $J_{\mathrm{d} \text {,in }}$ due to the advance of $R_{\mathrm{cr}}$ and the resulting addition of a new mass within this radius on nearly circular orbits. Next, we relate the motion of $R_{\mathrm{cr}}$ to the slowdown of the bar, $d R_{\mathrm{cr}} / d t \equiv \dot{R}_{\mathrm{cr}}=-R_{\mathrm{cr}} \dot{\Omega}_{\mathrm{b}} / \Omega_{\mathrm{b}}$. The torque on the bar gives the rate of change of its angular momentum (and, therefore, change in the angular momentum of the inner

5 In fact, we can impose this condition already from $t=0$, as variation of $J_{\mathrm{d} \text {,in }}$ is relatively small during the buckling (e.g., Fig. 9) 
disk):

$$
T=\frac{d}{d t}\left(I_{\mathrm{b}} \Omega_{\mathrm{b}}+J_{\text {circ }}\right),
$$

where $I_{\mathrm{b}}$ is the moment of inertia of the bar and $J_{\text {circ }}$ is the angular momentum of the internal circulation within the bar. (The second term does not appear in eq. 10 of Athanassoula (2003).) Hence, $T=\dot{\Omega}_{\mathrm{b}} I_{\mathrm{b}}+\Omega_{\mathrm{b}} \dot{I}_{\mathrm{b}}+\dot{J}_{\text {circ }}$. Assuming that the dominant loss of the angular momentum by the bar is due to the slowdown of its tumbling $\dot{\Omega}_{\mathrm{b}}$ (not a trivial assumption and definitely not a general one!), we can re-write eq. 5, replacing its first term by the action of $T$, as

$$
\dot{\Omega}_{\mathrm{b}} I_{\mathrm{b}}-v_{\mathrm{c}} R_{\mathrm{cr}}^{3} \Sigma_{\mathrm{cr}} \frac{\dot{\Omega}_{\mathrm{b}}}{\Omega_{\mathrm{b}}} \approx \dot{\Omega}_{\mathrm{b}}\left(I_{\mathrm{b}}-R_{\mathrm{cr}}^{2} M_{\mathrm{b}}\right)=0,
$$

where we have taken $M_{\mathrm{b}} \sim R_{\mathrm{cr}}^{2} \Sigma_{\mathrm{cr}}$. If the second term in eq. 7 loosely represents the moment of inertia of the bar, the net change in $J_{\mathrm{d} \text {,in }}$ is indeed negligible, explaining its near constancy in Fig. 8 (mid column) up to the time when $R_{\text {cr }}$ leaves the disk. In summary, we show that the constancy of the angular momentum within $R_{\mathrm{cr}}$ can be indeed explained if a number of straightforward assumptions is made to allow for an analytical estimates. These assumptions are as following: (1) the bar extends to near $R_{\mathrm{cr}}$ during this time and the disk mass within this radius lies mainly in the bar; $(2)$ the rate of change of the angular momentum in the bar due to the change in its moment of inertia and internal circulation is smaller than that resulting from the bar slowdown; (3) the circular velocity in the disk around the $R_{\mathrm{cr}}$ region and beyond is independent of radius; and (4) the average tangential velocity of the material in the bar is a fixed fraction of the disk circular velocity. While this behavior shows up in all our models, as long as $R_{\text {cr }}$ lies within the disk, a more general set of mass distributions should be tested before making a final conclusion.

While the halo absorbs all $J$ emitted by the disk, it is instructive to look into which parts of the halo are especially active in this process. Figure 9 displays the evolution of the absorbed $J_{\mathrm{h} \text {,in }}$ and $J_{\mathrm{h} \text {,out }}$. We observe that the inner halo becomes more receptive to the process with time, except in M40 and T16. This is clearly related to the outward motion of $R_{\mathrm{cr}}$. We note also, that the rate of $J$ absorption by the inner halo increases substantially after $R_{\mathrm{cr}} / R_{\mathrm{d}} \sim 1$, and the halo serves as the only sink of the angular momentum. Asymptotically, one can divide the models in two classes: when the rate of $J$ absorption by the inner halo goes to zero or even becomes negative (e.g., M70, T22, MT1), or when it declines only slightly (e.g., SD, M40, C30).

An alternative way to detail the exchange of the angular momentum in the disk-halo system is to divide the disk and halo into a number of concentric cylindrical shells. We have constructed a 2-D map of the angular momentum in each shell as a function of radius and time. It is more revealing, however, to plot the time derivative of $J_{\mathrm{d}}$ and $J_{\mathrm{h}}$ at each radius. This analysis was performed separately for the halo and the disk. In Figures 10 to 14 . we display color-coded diagrams of the rate of change of angular momentum $\dot{J}_{d, R}=\left(\partial J_{\mathrm{d}} / \partial t\right)_{\mathrm{R}}$ with radius and time for each run, and the same for the halo. Red color corresponds to emission and blue to absorption of the angular momentum. All the diagrams corresponding to

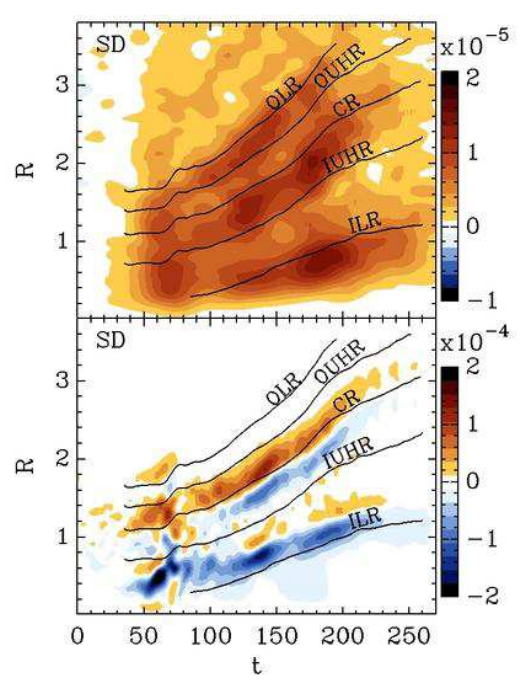

FIG. 10. - The rate of angular momentum flow $\dot{J}$ as a function of radius and time for the $\mathrm{SD}$ model (see also the text). The top row corresponds to the halo and the bottom row to the disk. Red/blue colors represent the absorption/emission of $J$ using a linear scale in color. The main resonances are indicated by the solid lines: the outer/inner Lindblad resonances (OLR/ILR), the outer/inner ultraharmonic resonances (OUHR/IUHR) and the corotation (CR). The cylindrical shells have $\Delta R=0.1$ and $z= \pm 2$. Time smearing is $\Delta t=3$.

disks have been calibrated identically. DM halos have also been calibrated uniformly among themselves.

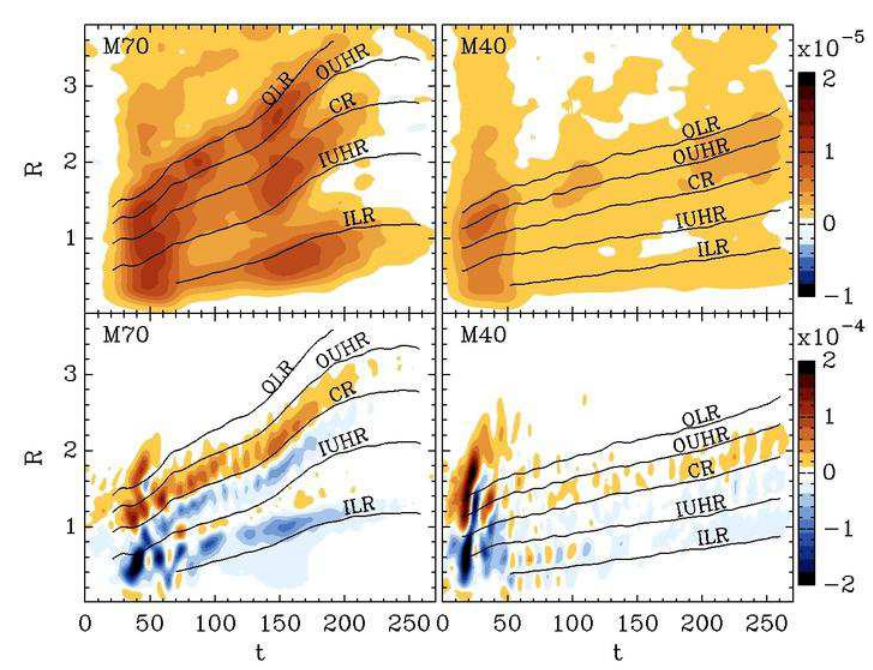

FIG. $11 .-\dot{J}$ as a function of radius and time for models M70 (left column) and M40 (right column). The top row corresponds to the halo and the bottom row to the disk. Color code and lines as in Fig. 10

The most striking features in Figures 10 to 14 is the continuity of color, i.e., emission and absorption bands, both in the disk and the halo, and their outward shift. The bands appear nonuniform in time and space. The halos are almost exclusively absorbers of the angular momentum, while the disks show regions of absorption and emission. From the halo evolution in the SD model 


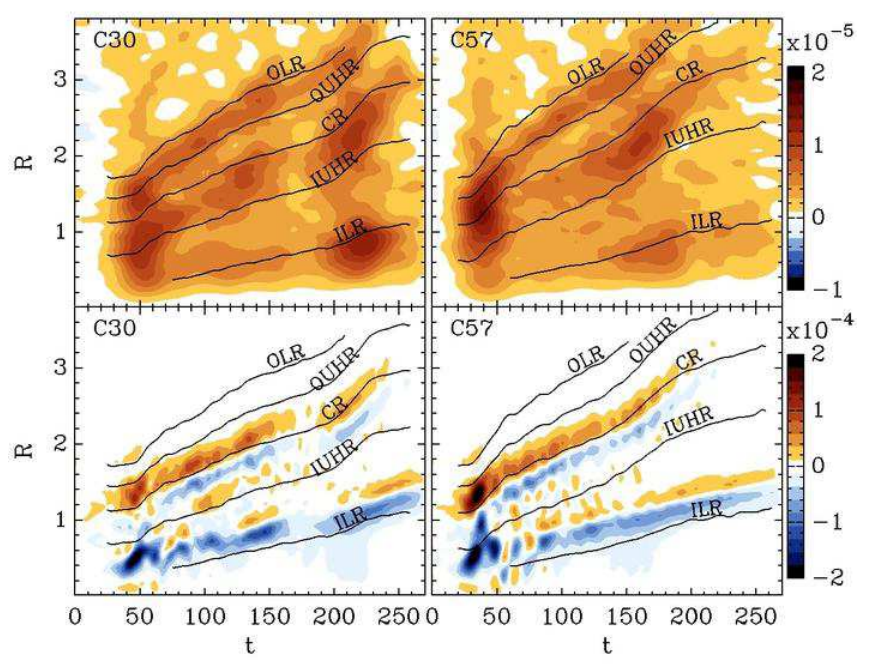

Fig. 12. $-\dot{J}$ as a function of radius and time for models C30 (left column) and C57 (right column). The top row corresponds to the halo and the bottom row to the disk. Color code and lines as in Fig. 10

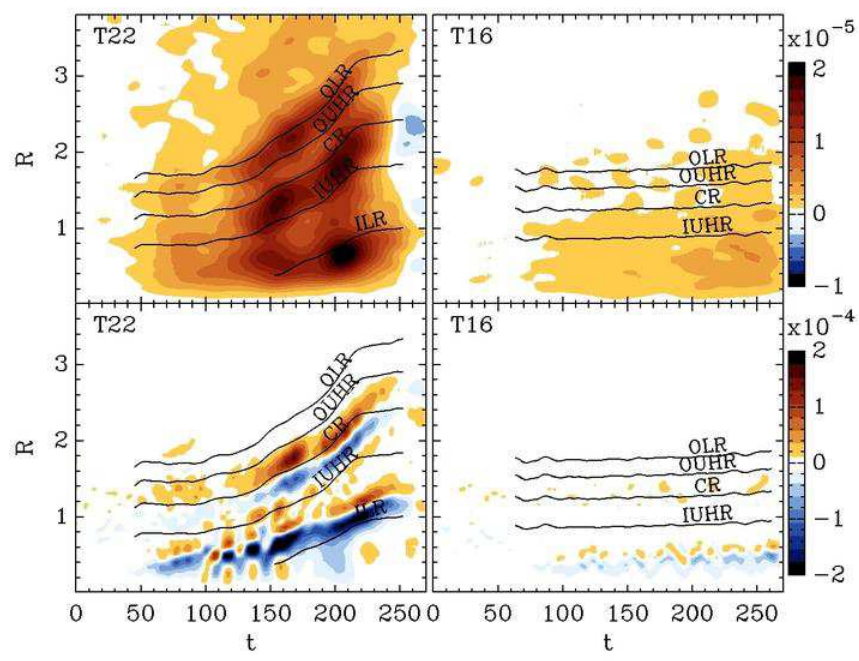

FIG. 13. $-\dot{J}$ as a function of radius and time for models T22 (left column) and T16 (rigt column). The top row corresponds to the halo and the bottom row to the disk. Color code and lines as in Fig. 10

(Fig. 11), we note that $J_{\mathrm{h}}$ is absorbed preferentially at three distinct bands which shift outwards with time. Other and weaker absorbing regions are observed between them and at larger radii. The absorption/emission bands in the disk are alternating, unlike in the halo.

To relate the emission/absorption bands to dynamical characteristics of the disk-halo system, we specify the positions of linear resonances on the color map. These are used for guidance purpose only, as the positions of linear resonances maybe misleading, especially for the strong bars, and especially for the inner Lindblad resonance(s).

We find a close correspondence between the behavior of $\dot{J}_{\mathrm{d}}(\mathrm{R})$ bands and the location of the linear resonances (superposed solid lines), namely, the corotation,

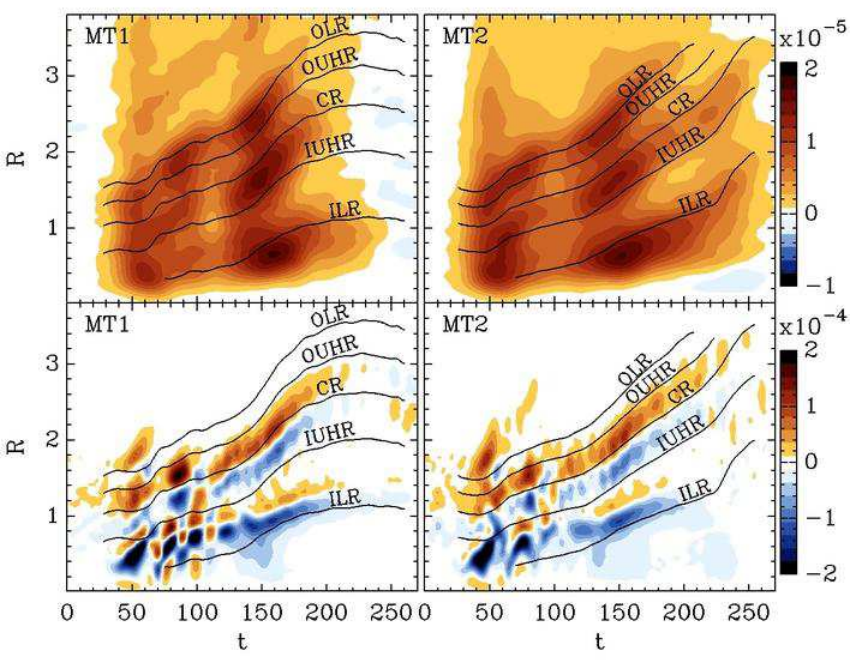

Fig. $14 .-\dot{J}$ as a function of radius and time for models MT1 (left column) and MT2 (right column). The top row corresponds to the halo and the bottom row to the disk. Color code and lines as in Fig. 10]

inner/outer Lindblad resonances (ILR/OLR), and the inner/outer ultra-harmonic resonances (UHR). While it is clear that the redistribution of $J$ in the disk and the halo is a strongly nonlinear process, some general conclusions can be made.

First, as expected, the emission and absorption between the inner disk and the inner halo components strongly correlate, with a certain interference of the outer disk (Figs. 11 14). Here we refer to inner/outer with respect to the $R_{\mathrm{cr}}$. The peak emission of $J$ by the disk happens at the band associated with the ILR. A weaker emission band lies at the inner UHR (see also Fig. 10 of Martinez-Valpuesta et al. 2006). The strongest absorption in the disk lies at the corotation and extends to the outer UHR. Weaker absorption is associated with the OLR in the disk. The halo absorption bands are centered on the ILR, inner UHR/corotation and the outer UHR/OLR. However, it is nonnegligible in other regions as well. We note that producing horizontal slices in the halo reveals that the lower halos, i.e., $\Delta z= \pm 0.3$, absorb mostly at the ILR while the upper halo absorb mostly at $R_{\mathrm{cr}}$. This is a refinement to previous results which shown that the main absorption is at the CR resonance (Athanassoula 2002; Martinez-Valpuesta et al. 2006).

Second, we observe temporal correlations between the emission and absorption peaks in the disk and the halo. Over the length of the run, both the disk and the halo experience from two to three 'times of activity,' except T160. The first such activity time is clearly associated with the first peak in $A_{2}$ and the subsequent buckling. The other activity time lies toward the saturation of $A_{2}$, late in the evolution of the bar. Some models, like SD, show an additional activity in between. This seems to be associated with $R_{\mathrm{cr}}$ crossing $R_{\mathrm{d}}$ - the halo absorption peaks at the corotation during this time. The resonances move outwards faster during intense $J$ absorption. The outward migration of the resonances is of course related to the bar slowdown, resulting in the resonances sweeping 
across the disk and halo material.

Third, $\dot{J}_{\mathrm{d}}(\mathrm{R})$ and $\dot{J}_{\mathrm{h}}(\mathrm{R})$ in Figs. 11,14 appear to correlate with $J_{\mathrm{d}, \text { in }}, J_{\mathrm{d} \text {,out }}, J_{\mathrm{h}, \text { in }}$ and $J_{\mathrm{h} \text {,out }}$ in Figs. 8 and 9] It also correlates with $A_{2}$ and especially with $A_{2 \mathrm{~b}}$. Lastly, the maxima of disk emission correspond to the maxima in the halo absorption.

Next, we return to the atypical behavior of some stellar bars in their secular phase, which exhibit $\Omega_{\mathrm{b}} \approx$ const., discussed in $\S 3.1$. In all the models where we observe this phenomenon, the ratio $R_{\mathrm{cr}} / R_{\mathrm{d}}>1$ at the time of detection. This behavior, as expected, is associated with the rate of $J$ transfer from the disk to the halo: in all these models, $\dot{J} \rightarrow 0$, both in the halo absorption or disk emission during $\Omega_{\mathrm{b}} \approx$ const. (Figs. 11 to 14). The outward motion of the disk resonances stalls as well (Figs. 5 and 11 - 14). Moreover, there is an indication that this is concurrent with the reversal of $J$ flow: while the disk still shows a weak emission at its ILR, the halo shows a weak emission at $R_{\mathrm{cr}}$ and a strong absorption at the ILR. The latter remains the most active resonance in the halo. The emission of $J$ by the halo at $R_{\text {cr }}$ is rather unusual and has not been observed before in any models in the literature, to the best of our knowledge.

Lastly, the disk inspection reveals the presence of two trailing spiral arms extending to $R_{\mathrm{cr}}$, and when $R_{\mathrm{cr}} / R_{\mathrm{d}}>1$, connecting the outer edge with $R_{\mathrm{cr}}$ (see $\S 3.3)$. Some stellar particles appear to be trapped at $R_{\mathrm{cr}}$ in its outward motion up to $t \sim 250$, depending on the model. These particles appear to concentrate at specific azimuthal locations of the CR "circle" with respect to the bar (in the bar frame of reference).

The particles in the spirals are not trapped. These spirals are prominent in Figs. 11 14, and are responsible for emission at $R_{\text {cr }}$ and absorption of $J$ in the IUHR-CR band, as $R_{\mathrm{cr}}$ moves outside the disk. While the number of these particles is not large, their specific angular momenta is the largest in the disk.

\section{DISCUSSION: BAR-DISK-HALO CORRELATIONS}

We have constructed and evolved three one-parameter and one two-parameter sequences of DM halos hosting a standard asymmetric stellar disk. All models developed bars and we have followed their evolution over a Hubble time. The properties of the bars, such as their strength, pattern speed, size, corotation radius, ratios of corotation to bar lengths, and more, have been related to those of the disks and host halos. Based on our results, we now test a number of correlations found by Athanassoula (2003). We also obtain additional ones - all of these will be used in order to compare with the gas models (Paper II).

For this purpose, we deal with three entities: the inner, bar-forming disk (defined within $R_{\mathrm{cr}}$ ), the disk as a whole, and the DM halo. We adopt the halo-to-disk mass ratio, $M_{\mathrm{h}} / M_{\mathrm{d}}$ within the disk velocity turnover radius, $R_{\text {turn }}=2.2 h$ (e.g., Sackett 1997) at $t=0$, as an independent variable, where $h$ is the disk radial scalelength defined in section 2.1. In the following, we also use the radial and vertical dispersion velocities in the disk and radial dispersions in the halo, and test how the efficiency of $J$ redistribution within the system depends on these parameters.

To quantify the role of the halo in the bar instability, we calculated the exponential timescale of $A_{2}$ growth,

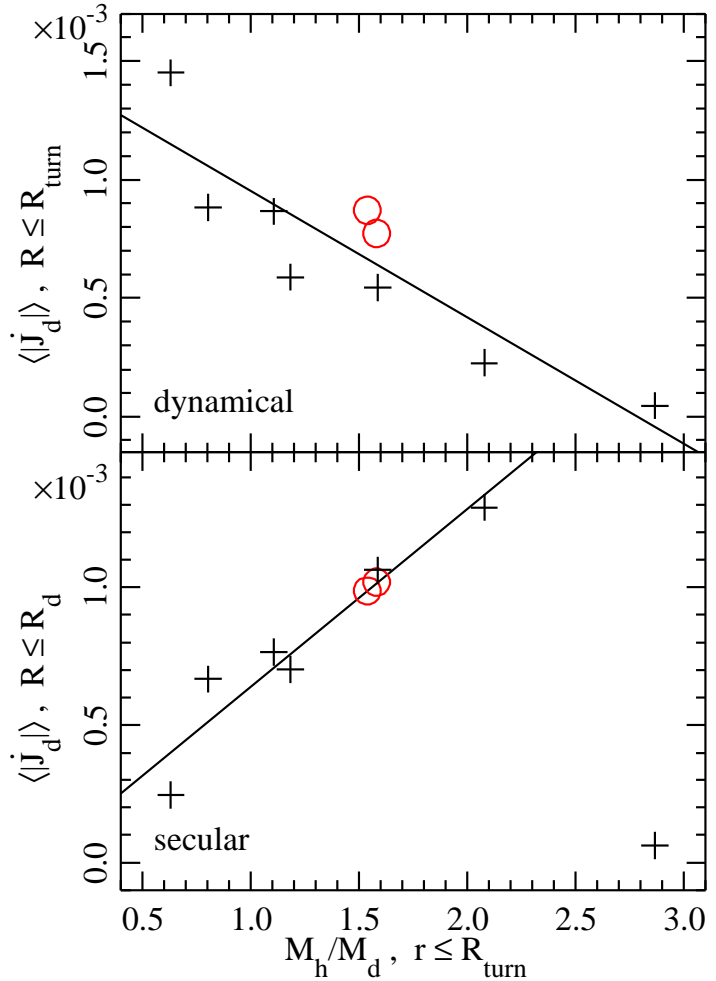

FIG. 15. - Average rate of angular momentum loss, $\langle|\dot{J}|\rangle$, by the inner stellar disk during its dynamical (upper) and secular (lower) phases of the bar evolution as a function of the $M_{\mathrm{h}} / M_{\mathrm{d}}$ ratio. The rate $\langle\dot{J}\rangle$ has been calculated within $R_{\text {turn }}$ (upper) and within $R_{\mathrm{d}}$ (lower). The mass ratio has been calculated within $R_{\text {turn }}$. The averaging was performed over the time period from $t=0$ to the end of an exponential growth of the stellar bars (upper) and from the time of the first minimum of $A_{2}$, i.e., the of the buckling, to its saturation, $t=213$ (about $10 \mathrm{Gyr}$ ) (lower). The green circles represent the two hybrid models. The attempted linear fit in the lower frame does not include the lower right corner point (T16).

$t_{\text {bar }}$, against $M_{\mathrm{h}} / M_{\mathrm{d}}$ within $R_{\text {turn }}$, which represents the halo concentration, normalized by the disk mass within the same radius. A linear correlation was obtained, namely, that an increase in the DM mass concentration leads to a slower bar growth before the first buckling. However, the bar evolution in the secular phase cannot be described by an exponential growth. Looking for a more universal measure of the bar growth at all times, we decided in favor of angular momentum change in the inner and full disks. Figure 15 displays the average rate of angular momentum change, $\langle|\dot{J}|\rangle$, in the dynamical and secular phases of the bar evolution, within $R_{\text {turn }}$ and $R_{\mathrm{d}}$ respectively. The former choice of a fixed radius $\left(R_{\mathrm{turn}}\right)$ results from total $J_{\mathrm{d}}$ being nearly unchanged before the buckling - $J$ is exchanged predominantly between the inner and outer disks across $R_{\mathrm{cr}}$ at this stage. We have refrained from using $R_{\text {cr }}$ as an inner/outer disk separator here because its outward motion brings in fresh, high $J / E$ material which 'contaminates' the $J$ transfer due to the resonant and non-resonant interactions between the inner and outer disks. The latter choice of $R_{\mathrm{d}}$ in the secular phase results from $J_{\mathrm{d} \text {,in }}$ being nearly constant $J$ is mostly exchanged between the disk and the halo. 


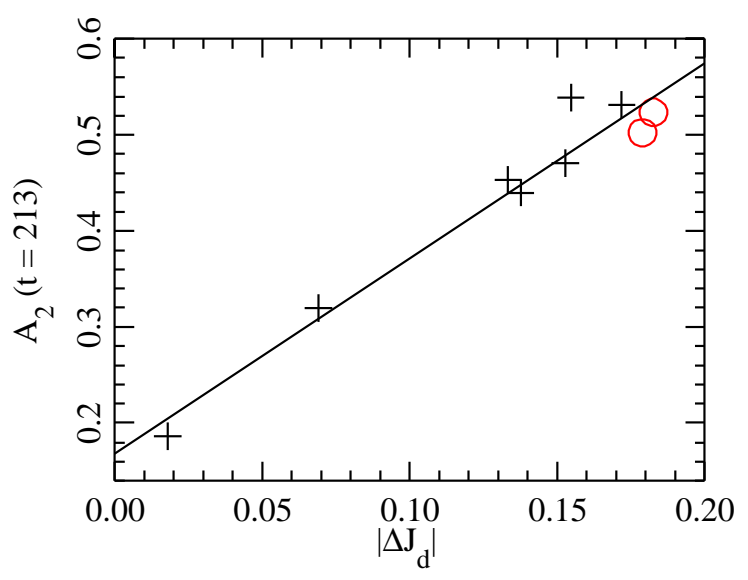

FIG. 16. - $A_{2}$ at $t \sim 213$, corresponding to $10 \mathrm{Gyr}$, as a function of the angular momentum change in the whole disk over $\Delta t=213$. The green circles represent the two hybrid models.

Figure [15] shows that clear correlations of $\langle|\dot{J}|\rangle$ with $M_{\mathrm{h}} / M_{\mathrm{d}}$ persist over the Hubble time - more concentrated halos provide an increasingly efficient damping of the bar instability by reducing the rate of $J$ transfer to the outer disk (upper frame). They do facilitate such a transfer in the secular phase (lower frame). Significantly, these correlations extend down at least to $M_{\mathrm{h}} / M_{\mathrm{d}} \sim 0.5$ - the disk-dominated models. We note that T16 exhibits a qualitatively and quantitatively different behavior and was not included in the attempted linear fit to the secular evolution (lower frame). We have discussed this model in $\S 3.4$.

The explanation for such a dual role of a DM halo in the bar evolution lies in determining what serves as a sink of $J_{\mathrm{d}, \text { in }}$ from the inner disk. As shown in $\S 3.6$, the outer disk beyond $R_{\mathrm{cr}}$ absorbs nearly all of $J$ during the bar instability. A more concentrated halo during this phase will dilute the disk gravity and, therefore, will act against the resonant orbit coupling (i.e., resonant torques) between the inner and outer disks. On the other hand, during the secular phase of the bar instability, after the first buckling, the halo serves as the sink of $J$ from the disk. In this case, if increase of the DM mass density leads to a concurrent increase in the DM phase space density near the resonances, it facilitates the bar-halo resonance coupling, unless counterbalanced by a 'hotter' halo. The $\langle\dot{J}\rangle$ rates appear similar during the dynamical and secular phases. But the duration of each phase can differ considerably. Therefore, one expects that the amount of $J$ acquired by the halo will be much more substantial during the secular phase compared to the angular momentum lost by the inner disk (within a fixed radius!) during the dynamical phase.

It is known that the DM halo affects the bar evolution. In particular, it was shown that the DM halo concentration anti-correlates with the bar growth time. Athanassoula (2003) has also demonstrated the (anti)-correlation between $\Omega_{\mathrm{b}}$ and $A_{2}$. Our Figure 15 quantifies the dual role played by the DM halo during the bar evolution and ties this explicitly to the rate of the angular momentum transfer within the disk-halo dynamical system.

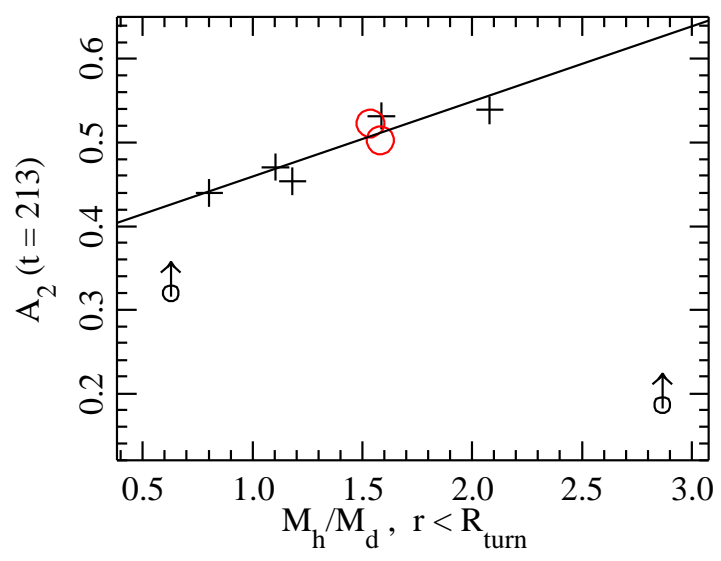

Fig. 17.- Saturated $A_{2}$ at $t=213(\sim 10 \mathrm{Gyr})$, as a function of the halo-to-disk mass ratio within $R_{\mathrm{turn}}$ at $t=0$. Two models do not reach the saturation and are shown as lower limits: M40 (left) and T16 (right). The green circles represent the two hybrid models.

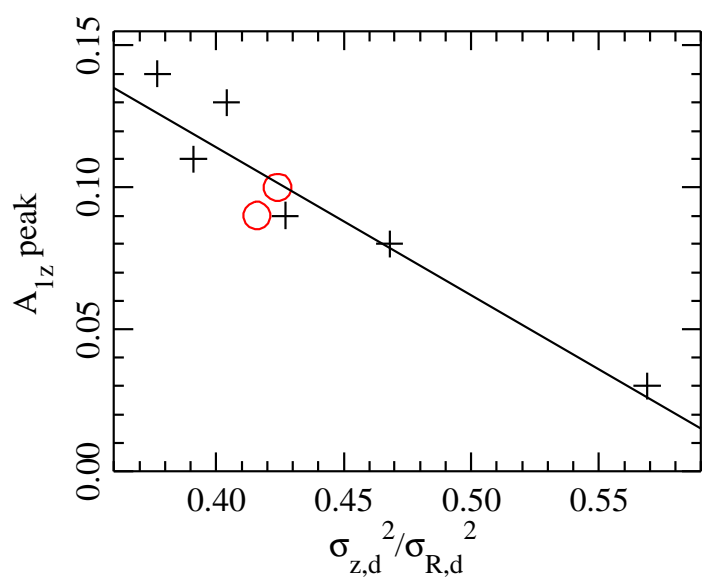

Fig. 18.- Dependence of $A_{1, \mathrm{z}}$ amplitude on the ratio of verticalto-radial velocity dispersions, $\left(\sigma_{\mathrm{z}, \mathrm{d}} / \sigma_{\mathrm{r}, \mathrm{d}}\right)^{2}$. T16 is excluded as it does not buckle. The green circles represent the two hybrid models. These velocity dispersions have been measured in a ring defined by $0.1 \leq R \leq 0.2$ and $|z| \leq 0.1$.

Stronger bars are expected to be more efficient in redistribution of the angular momentum in the disk-halo systems. In our collisionless models, bars strengthen monotonically only prior to the first peak and after the first buckling. Despite the overall non-monotonic behavior of the bar, its final strength correlates with the amount of $\Delta J_{\mathrm{d}}$ lost by the disk and acquired by the halo (Fig. 16), in agreement with Athanassoula (2003).

For the same reason, we expect and obtain correlation between $A_{2}$ at $t=213(\sim 10 \mathrm{Gyr})$, and $M_{\mathrm{h}} / M_{\mathrm{d}}$ ratio within $R_{\text {turn }}$, i.e., the halo mass concentration (Fig. 17), thus confirming Athanassoula (2003) claim. By this time, nearly all modeled bar strengths reach saturation (e.g., Fig. 31), except in two models, M40 and T16, shown as lower limits. As shown in $\S 3.3, A_{2}$ and $A_{2 \mathrm{~b}}$ saturate shortly after $R_{\mathrm{cr}} / R_{\mathrm{d}} \sim 1$. These models represent two 


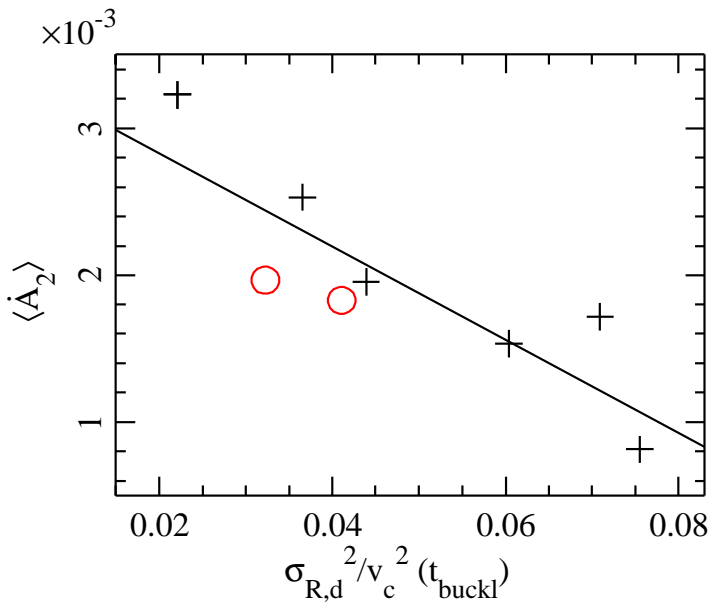

FIG. 19.- Secular evolution of the bars: dependence of $\left\langle\dot{A}_{2}\right\rangle$, the average growth rate of the bar, between $t_{\text {buckl }}$ and $t=213$ (10 Gyr), vs $\sigma_{\mathrm{r}, \mathrm{d}} / v_{\mathrm{c}}$, the ratio of the radial dispersion in the disk to the circular velocity at $R_{\text {turn }}$. The latter ratio is calculated at $t_{\text {buckl. }}$. T16 is omitted as it does not buckle. The green circles represent the two hybrid models.

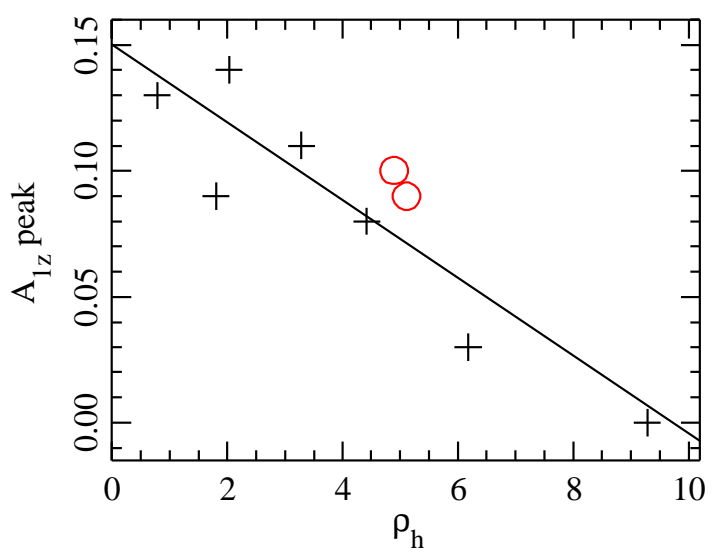

Fig. 20.- Dependence of $A_{1, \mathrm{z}}$ peak on the central halo density at $t=0$. The green circles represent the two hybrid models.

extreme trends in the halo mass concentration. T16 has the most concentrated halo, does not buckle and its secular evolution differs from other bars. M40 represents the least concentrated halo with a fractionally most massive disk (Figs. 1 and 2). It has the shortest exponential growth for the bar, $t_{\text {bar }} \sim 10$. Therefore, it is puzzling why its bar strength does not saturate. While M40 exhibits buckling and subsequent drop in $A_{2}$ and $A_{2 \mathrm{~b}}$, its secular growth is the slowest of all models and comparable to that of T16. Its bar is the longest of all models as the ansae do not disappear. Furthermore, its $R_{\mathrm{cr}}$ lies always within the disk, while $R_{\mathrm{cr}} / R_{\mathrm{b}} \sim 1.2$ and remains flat for a Hubble time. All these properties appear unique in our models.

While the behavior of T16 is partially understandable, that of M40 requires more explanation. It is difficult to explain within the context of the halo mass concentration only, and seems to require additional parameter.
We invoke the disk radial dispersion velocity, $\sigma_{\mathrm{R}, \mathrm{d}}$, which in a way also governs the bar vertical buckling instability through its ratio to the vertical velocity dispersion, $\sigma_{\mathrm{z}, \mathrm{d}}$. We test this idea by first plotting the amplitude of buckling, $A_{1, \mathrm{z}}$, vs this ratio calculated just before the first buckling (Fig. 18). Indeed, M40 has the smallest $\left(\sigma_{\mathrm{z}, \mathrm{d}} / \sigma_{\mathrm{R}, \mathrm{d}}\right)^{2}$ among our models, and, therefore, is expected and, in fact, displays the largest buckling amplitude $A_{1, \mathrm{z}}$ (Fig. 6). A side effect of this buckling is the overall heating of the disk. Consequently, the disk will be less susceptible to the bar growth in the secular phase. Fig. 19] shows the growthrate of the bar strength averaged over the secular phase, $\left\langle\dot{A}_{2}\right\rangle$, i.e., between the buckling time, $t_{\mathrm{buckl}}$, and the saturation time, $t \sim 213(\sim 10 \mathrm{Gyr})$, vs $\sigma_{\mathrm{R}, \mathrm{d}} / v_{\mathrm{c}}$ measured at $t_{\mathrm{buckl}}$ (i.e., at the minimum of $\left.A_{2}\right)$. A clear correlation exists between these parameters - 'hotter' disk impairs the bar growth in the secular phase of its evolution. Note that M40 has the hottest disk in this stage, which would explain its anomalously slow bar growth. The same correlation against $\sigma_{\mathrm{R}, \mathrm{d}}$ looks weaker and shows much larger dispersion of individual models.

As mentioned before, in each separate sequence, the $A_{1, \mathrm{z}}$ peak is stronger for models progressively less dominated by the halo. It is interesting that an anticorrelation between the initial central density of the halo and the strength of the first $A_{1, \mathrm{z}}$ peak holds for our models altogether, regardless of which halo parameter is varied (Figure 20). Moreover, the anticorrelation exists between the strength of the first $A_{1, \mathrm{z}}$ peak and the ratio of the vertical-to-radial dispersion velocities, shown in Figure 18.

To summarize, we confirm the previous results and quantify for the first time the dual role that the DM halos play in stellar bar evolution (Fig. 15): more centrally concentrated halos slowdown dynamical processes in the disk, such as spontaneous bar instability and vertical buckling instability, as well as angular momentum redistribution in the system. They reverse this trend and facilitate the angular momentum transfer during the secular stage of bar evolution, following the buckling. We follow the angular transfer in the disk-halo systems by varying one basic parameter at the time in order to identify the sites and times of intense angular momentum flows. While we confirm the earlier works which have identified the ILR and corotation resonances as being primarily responsible for $J$ emission by the disk and absorption by the halo, we also find few caveats.

First, the total angular momentum in the disk is largely conserved before the first buckling, thus $J$ flows across the corotation to the outer disk, although the amount is relatively low compared to the subsequent exchange. Second, during the secular stage of the bar evolution, the angular momentum within the corotation resonance is largely conserved: the loss of $J$ by this region due to the gravitational torques on the bar (resonant and non-resonant) is compensated by the influx of new material, rich in $J$, across the corotation, due to its outward motion. We also elaborate under what conditions this 'conspiracy' law operates. In view of this, the ratio of $R_{\mathrm{cr}} / R_{\mathrm{d}}$ emerges as important dynamic discriminator between various paths in barred disk evolution — its value can be determined if bar pattern speeds are known. 
Third, we find that in some models the bar pattern speed stalls becoming nearly constant for prolonged time periods. All stellar bars which show this behavior have their corotation lying outside the disk. The disk-halo angular momentum exchange nearly vanishes and the halos display a weak emission of $J$ at the corotation and $a b$ sorption at the ILR. While otherwise the bar pattern speeds are generally strongly decaying over the secular timescale, the addition of the gas component can modify this trend and soften the bar braking. We postpone our conclusions on this issue to Paper II. We also find that $R_{\mathrm{cr}} / R_{\mathrm{b}}$ ratio stays within $1-1.4$ range, only occasionally spiking above it to $\sim 1.8$. This behavior is typical after the first buckling and as long as $R_{\mathrm{cr}} / R_{\mathrm{d}} \lesssim 1$.

Lastly, we confirm some known correlations (Figs. 16 and 17) between the basic parameters of the disk-halo system, e.g., between the final bar strength and $M_{\mathrm{h}} / M_{\mathrm{d}}$ ratio. Model M40 with the least concentrated halo does not follow this trend and we test the possibility that an additional parameter plays the role in this, i.e., the dispersion velocities ratio $\sigma_{\mathrm{z}, \mathrm{d}} / \sigma_{\mathrm{R}, \mathrm{d}}$. Because M40 experiences the strongest buckling, the disk is heated up, which impairs the subsequent bar growth and can explain the behavior of M40 in the previous correlation. Figs. 18, 19 and 20 display new correlations between various parameters. The bar average growthrate over its secular evolution time decreases with increasing dispersion velocities in the disk, $v_{\mathrm{R}, \mathrm{d}} / v_{\mathrm{c}}$. We also show that in a closely associated relation - the angular momentum lost by the disk, $\Delta J_{\mathrm{d}}$, over the evolution correlates with the final bar strength. Finally, the amplitude of the first buckling depends on the central density in the DM halo. These correlations will be followed up when the gas component is present. We expect substantial modifications in the relations between various basic parameters in the disk-halo system.

We are grateful to Ingo Berentzen for comments on the manuscript, and to Ron Buta for answering our questions. We also thank Ingo Berentzen and Inma MartinezValpuesta for help with numerical issues. This research has been partially supported by NASA/LTSA/ATP, NSF and STScI grants to I.S. STScI is operated by the AURA, Inc., under NASA contract NAS 5-26555. I.S. is grateful to the JILA Fellows for support.

\section{REFERENCES}

Athanassoula. E. \& Sellwood, J.A. 1986, MNRAS, 221, 213

Athanassoula, E. 1992, MNRAS, 259, 328

Athanassoula, E. \& Misiriotis, A. 2002, MNRAS, 330, 35

Athanassoula, E. 2002, ApJ, 569, L83

Athanassoula, E. 2003, MNRAS, 341, 1179

Athanassoula, E. 2008, MNRAS, 390, L69

Berentzen, I., Shlosman, I., Martinez-Valpuesta, I. \& Heller, C.H. 2007, ApJ, 666, 189

Ceverino, D. \& Klypin, A. 2007, MNRAS, 379, 1155

Christodoulou, D.M., Shlosman, I. \& Tohline, J.E. 1995a, ApJ, 443,551

Christodoulou, D.M., Shlosman, I. \& Tohline, J.E. 1995b, ApJ, 443,563

Combes, F. \& Sanders, R.H. 1981, A\&A, 96, 164

Combes, F., Debbasch, F., Friedli, D. \& Pfenniger, D. 1990, A\&A, 233,82

Debattista, V.P. \& Sellwood, J.A. 2000, ApJ, 543, 704

Dehnen, W. 2002, J. Comput. Phys., 179, 27

Dubinski, J., Berentzen, I. \& Shlosman, I. 2009, ApJ, 697, 293

Efstathiou, G., Lake, G. \& Negroponte, J. 1982, MNRAS, 199, 1069

Friedli, D. \& Pfenniger, D. 1990, ESO/CTIO Workshop on Bulges of Galaxies, ed. B. Jarvis \& D.M. Terndrup (Garching: ESO), 265

Heller, C.H. \& Shlosman, I. 1994, ApJ, 424, 84

Heller, C.H., Shlosman, I. \& Athanassoula, E. 2007a, ApJ, 657, L65

Heller, C.H., Shlosman, I. \& Athanassoula, E. 2007b, ApJ, 671 226
Hernquist, L. 1993, ApJ, 356, 359

Kormendy, J. \& Kennicutt, R.C. 2004, ARAA, 42, 603

Lynden-Bell, D. \& Kalnajs, A.J. 1972, MNRAS, 157, L1

Martinez-Valpuesta, I. \& Shlosman, I. 2004, ApJ, 613, L29

Martinez-Valpuesta, I. 2006, Ph.D. Thesis, University of Hertsfordshire

Martinez-Valpuesta, I., Shlosman, I. \& Heller, C.H. 2006, ApJ, 637, 214

Masset, F. \& Tagger, M. 1997, A\&A, 322, 422

Ostriker, J.P. \& Peebles, P.J.E. 1973, ApJ, 186, 467

Pfenniger, D. \& Friedli, D. 1991, A\&A, 252, 75

Raha, N., Sellwood, J.A., James, R.A. \& Kahn, F.D. 1991, Nature, 352,411

Romano-Diaz, E., Shlosman, I., Heller, C.H. \& Hoffman, Y. 2008, ApJ, 687, L13

Sackett, P.D. 1997, ApJ, 483, 103

Sellwood, J.A. 1980, A\&A, 89, 296

Toomre A. 1981, The Structure and Evolution of Normal Galaxies, CUP: Cambridge, Fall S. M. \& Lynden-Bell D. (eds.), 111

Tremaine, S. \& Weinberg, M.D. 1984, MNRAS, 209, 729

Valenzuela, O. \& Klypin, A. 2003, MNRAS, 345, 406

van Albada, T.S. \& Sanders, R.H. 1982, MNRAS, 201, 303

Weinberg, M.D. 1985, MNRAS, 213, 451

Weinberg, M.D. \& Katz, 2007a, MNRAS, 375, 425

Weinberg, M.D. \& Katz, 2007b, MNRAS, 375, 460 